TRANSACTIONS OF THE

AMERICAN MATHEMATICAL SOCIETY

Volume 361, Number 11, November 2009, Pages 5753-5780

S 0002-9947(09)04654-6

Article electronically published on June 19, 2009

\title{
POISSON STRUCTURES ON AFFINE SPACES AND FLAG VARIETIES. II
}

\author{
K. R. GOODEARL AND M. YAKIMOV
}

Dedicated to the memory of our colleague Xu-Dong Liu (1962-2005)

\begin{abstract}
The standard Poisson structures on the flag varieties $G / P$ of a complex reductive algebraic group $G$ are investigated. It is shown that the orbits of symplectic leaves in $G / P$ under a fixed maximal torus of $G$ are smooth irreducible locally closed subvarieties of $G / P$, isomorphic to intersections of dual Schubert cells in the full flag variety $G / B$ of $G$, and their Zariski closures are explicitly computed. Two different proofs of the former result are presented. The first is in the framework of Poisson homogeneous spaces, and the second one uses an idea of weak splittings of surjective Poisson submersions, based on the notion of Poisson-Dirac submanifolds. For a parabolic subgroup $P$ with abelian unipotent radical (in which case $G / P$ is a Hermitian symmetric space of compact type), it is shown that all orbits of the standard Levi factor $L$ of $P$ on $G / P$ are complete Poisson subvarieties which are quotients of $L$, equipped with the standard Poisson structure. Moreover, it is proved that the Poisson structure on $G / P$ vanishes at all special base points for the $L$-orbits on $G / P$ constructed by Richardson, Röhrle, and Steinberg.
\end{abstract}

\section{INTRODUCTION}

In this paper, we investigate the geometry of the standard Poisson structures on flag varieties of complex reductive algebraic groups $G$. We prove that, in such flag varieties, there are only finitely many orbits of symplectic leaves with respect to a fixed maximal torus $H$ of $G$. These $H$-orbits are isomorphic to intersections of dual Schubert cells in the full flag variety of $G$, and their closures are explicitly determined. Further, these $H$-orbits are parametrized by explicit subsets of the double Weyl group of $G$, and inclusions of closures are determined by an explicit relation in terms of the Bruhat order. Additional structure is obtained in the case of Hermitian symmetric spaces of compact type, i.e., for flag varieties $G / P$ where $P$ is a parabolic subgroup of $G$ with abelian unipotent radical.

A more precise statement of our main results is given in Theorem 0.4 below. First, however, we need to fix some notation.

Received by the editors June 13, 2007.

2000 Mathematics Subject Classification. Primary 14M15; Secondary 53D17, 14L30, 17B20, 17B63, 53C35.

The research of the first author was partially supported by National Science Foundation grant DMS-0401558.

The research of the second author was partially supported by National Science Foundation grant DMS-0406057 and an Alfred P. Sloan research fellowship.

(C)2009 American Mathematical Society Reverts to public domain 28 years from publication 
0.1. Let $G$ be a connected reductive algebraic group over $\mathbb{C}$. Fix a pair of dual Borel subgroups $B^{ \pm}$in $G$, and set $H=B^{+} \cap B^{-}$for the corresponding maximal torus. Denote the corresponding Lie algebras by $\mathfrak{g}, \mathfrak{b}^{ \pm}$, and $\mathfrak{h}$. Denote by $\Delta$ and $\Delta^{+}$the set of all roots, respectively all positive roots, of $\mathfrak{g}$ with respect to $\mathfrak{h}$. Let $\Gamma$ be the set of all positive simple roots of $\mathfrak{g}$. For a subset $J$ of $\Gamma$, let $P_{J}^{ \pm}$be the standard parabolic subgroups of $G$, containing respectively the Borel subgroups $B^{ \pm}$. Let $L_{J}=P_{J}^{+} \cap P_{J}^{-}$be the common Levi factor of $P_{J}^{ \pm}$. Denote by $U^{ \pm}$and $U_{J}^{ \pm}$ the unipotent radicals of $B^{ \pm}$and $P_{J}^{ \pm}$, respectively. Set $\mathfrak{p}_{J}^{ \pm}=\operatorname{Lie} P_{J}^{ \pm}, \mathfrak{l}_{J}=\operatorname{Lie} L_{J}$, $\mathfrak{n}^{ \pm}=\operatorname{Lie} U^{ \pm}$, and $\mathfrak{n}_{J}^{ \pm}=\operatorname{Lie} U_{J}^{ \pm}$.

0.2. We fix a nondegenerate bilinear invariant form $\langle.,$.$\rangle on \mathfrak{g}$ for which the square of the length of a long root is equal to 2 . Recall [3, eq. (1.1)] that the standard $r$-matrix of $\mathfrak{g}$ is given by

$$
r_{\mathfrak{g}}=\sum_{\alpha \in \Delta_{+}} \frac{\langle\alpha, \alpha\rangle}{2} e_{\alpha} \wedge f_{\alpha}
$$

where $\left\{e_{\alpha}\right\}_{\alpha \in \Delta^{+}}$and $\left\{f_{\alpha}\right\}_{\alpha \in \Delta^{+}}$are any sets of root vectors of $\mathfrak{g}$, normalized by $\left[e_{\alpha}, f_{\alpha}\right]=\alpha^{\vee}=2 \alpha /\langle\alpha, \alpha\rangle$. (In the last equation, $\mathfrak{h}$ and $\mathfrak{h}^{*}$ are identified via the restriction of the form $\langle.,$.$\rangle ; for \alpha \in \Delta^{+}, e_{\alpha}$ and $f_{\alpha}$ are root vectors for the roots $\alpha$ and $-\alpha$, respectively.) The standard Poisson structure on $G$ is given by

$$
\pi_{G}=L\left(r_{\mathfrak{g}}\right)-R\left(r_{\mathfrak{g}}\right)=\chi^{R}\left(r_{\mathfrak{g}}\right)-\chi^{L}\left(r_{\mathfrak{g}}\right) .
$$

Here $L\left(r_{\mathfrak{g}}\right)$ and $R\left(r_{\mathfrak{g}}\right)$ refer to the left and right invariant bivector fields on $G$ associated to $r_{\mathfrak{g}} \in \bigwedge^{2} \mathfrak{g} \cong \bigwedge^{2} T_{e} G$.

For any subset $J \subseteq \Gamma$, the standard parabolic subgroup $P_{J}^{+}$is a Poisson algebraic subgroup of $\left(G, \pi_{\mathfrak{g}}\right)$. The natural projection

$$
\eta_{J}: G \rightarrow G / P_{J}^{+}
$$

induces the following Poisson structure on the flag variety $G / P_{J}^{+}$:

$$
\pi_{J}:=\eta_{J *}(\pi)=-\chi\left(r_{\mathfrak{g}}\right) ;
$$

see [3, Theorem 1.8] and Proposition 1.3 below. Throughout the paper, for an action of $G$ on a variety $M$, we denote the extension to $\wedge \mathfrak{g}$ of the infinitesimal action of $\mathfrak{g}$ on $M$ by

$$
\chi: \bigwedge \mathfrak{g} \rightarrow \Gamma(M, \bigwedge T M) .
$$

For brevity, we set $\eta=\eta_{\emptyset}$ and $\pi=\pi_{\emptyset}$.

Since the Poisson structure $\pi_{G}$ vanishes on $H$, the left action of $H$ on $G / P_{J}^{+}$ preserves $\pi_{J}$.

0.3. In this paper we investigate the geometry of the Poisson structures $\pi_{J}$ on the flag varieties $G / P_{J}^{+}$. This continues our work with Brown in [3, which we shall refer to as Part I.

Before we state the main results of the paper, we introduce some notation on Weyl groups. The Weyl group of the pair $(G, H)$ will be denoted by $W$. For $w \in W$, we will denote by $\dot{w}$ a representative of $w$ in the normalizer of $H$. If a formula does not depend on the choice of this representative the dot will be omitted. The Weyl group of $\left(L_{J}, H\right)$, naturally thought of as a subgroup of $W$, will be denoted by $W_{J}$. Recall that each coset in $W / W_{J}$ has unique minimal and maximal length representatives. The sets of those will be denoted by $W_{\min }^{J}$ and $W_{\max }^{J}$ respectively. Denote the longest elements of $W$ and $W_{J}$ by $w_{\circ}$ and $w_{\circ}^{J}$. 
For $w \in W$ and $J \subseteq \Gamma$, set

$$
x_{w}^{J}=w P_{J}^{+} \in G / P_{J}^{+}, \quad x_{w}=x_{w}^{\emptyset}=w B^{+} \in G / B^{+} .
$$

0.4. The following Theorem summarizes some of our results (Theorems 1.5, 1.8, 4.6, and Proposition 4.2). Recall from Part I, [3, eq. (2.11)], the notation

$$
\mathcal{U}_{\dot{w}_{1}, w_{2}}=U_{w_{1}}^{-} \dot{w}_{1} \cap B^{+} w_{2} B^{+}, \quad w_{1}, w_{2} \in W,
$$

where for $w \in W$,

$$
U_{w}^{-}=U^{-} \cap \operatorname{Ad}_{w}\left(U^{-}\right) .
$$

Theorem. (i) There are only finitely many $H$-orbits of symplectic leaves on $\left(G / P_{J}^{+}, \pi_{J}\right)$, parametrized by pairs $\left(w_{1}, w_{2}\right) \in W_{\max }^{J} \times W$ such that $w_{1} \leq w_{2}$ in the Bruhat order. The torus orbit corresponding to the pair $\left(w_{1}, w_{2}\right)$ is given by

$$
\mathcal{S}_{w_{1}, w_{2}}^{J}=\mathcal{U}_{\dot{w}_{1}, w_{2}} \cdot P_{J}^{+}
$$

and is biregularly isomorphic to the intersection $\mathcal{B}_{w_{1}, w_{2}}=B^{-} x_{w_{1}} \cap B^{+} x_{w_{2}}$ of dual Schubert cells in the generalized full flag variety $G / B^{+}$. Thus, the $H$-orbits of symplectic leaves on $\left(G / B^{+}, \pi\right)$ are exactly the intersections of dual Schubert cells $\mathcal{B}_{w_{1}, w_{2}}$.

(ii) For each pair $\left(w_{1}, w_{2}\right)$ as above, the Zariski closure of $\mathcal{S}_{w_{1}, w_{2}}^{J}$ in $\left(G / P_{J}^{+}, \pi_{J}\right)$ is equal to the union of $\mathcal{S}_{v_{1}, v_{2}}^{J}$ over those pairs $\left(v_{1}, v_{2}\right) \in W_{\max }^{J} \times W$ with $v_{1} \leq v_{2}$ for which there exists $z \in W_{J}$ such that $w_{1} \leq v_{1} z$ and $w_{2} \geq v_{2} z$.

(iii) If $P_{J}^{+}$is a parabolic subgroup of $G$ with abelian unipotent radical (in which case $G / P_{J}^{+}$is a Hermitian symmetric space of compact type), then all $L_{J}$-orbits on $G / P_{J}^{+}$are complete Poisson subvarieties of $\left(G / P_{J}^{+}, \pi_{J}\right)$, and all of them are quotients of the standard Poisson group structure on $L_{J}$.

In addition, we prove that in the case of Hermitian symmetric spaces of compact type the Poisson structure $\pi_{J}$ vanishes at the base points for the $L_{J}$-orbits constructed by Richardson, Röhrle, and Steinberg [19]. In this case, we also characterize explicitly the $H$-orbits of symplectic leaves which fall within a given $L_{J}$-orbit. This relates parts (i) and (iii) of the above Theorem. It is done in Theorem 4.13 and will not be formulated here due to the amount of notation it requires.

The partition of the partial flag variety $G / P_{J}^{+}$into $H$-orbits of leaves coincides with Lusztig's partition [14 of $G / P_{J}^{+}$into locally closed subvarieties which are isomorphic to intersections of dual Schubert cells in the full flag variety $G / B^{+}$. More precisely, $\mathcal{S}_{w_{1}, w_{2}}^{J}=\mathcal{P}_{w_{1}, w_{2}^{\prime}, w_{2}^{\prime \prime}}^{J}$ (in the notation of [20, Section 5]), where $w_{2}^{\prime}$ and $w_{2}^{\prime \prime}$ are the unique elements in $W_{\min }^{J}$ and $W_{J}$ such that $w_{2}=w_{2}^{\prime} w_{2}^{\prime \prime}$. This follows, e.g., from the discussion in Rietsch 20, Section 5] and $\S 1.5$ below.

We also derive explicit formulas for the restrictions of the Poisson structures $\pi_{J}$ to the open $B^{-}$-orbit in Hermitian symmetric spaces of compact type for classical groups and show that in all cases those are the quasiclassical limits of classes of quadratic algebras that attracted a lot of attention in the theory of quantum groups (see Section 5). While those classes were previously studied case by case, our work conceptually unifies them. Finally, in the exceptional case $E_{6}$ we find a new interesting quadratic Poisson structure on a 16 dimensional affine space, related to a half-spin representation of $\mathfrak{s o}_{10}$.

0.5. We offer two different proofs of the first part of Theorem 0.4. The first one, which appears in Section 1, uses the theory of Poisson homogeneous spaces. The 
second one (see Section 3) is more geometric and is based upon the notion of Poisson-Dirac submanifolds [26], [5]. The latter are submanifolds of a Poisson manifold $(M, \Pi)$ which in general might not be Poisson submanifolds but have the property that their symplectic leaves are exactly the connected components of their intersections with the symplectic leaves of $(M, \Pi)$. The second approach uses the idea of weak splittings of surjective Poisson submersions, developed in Section 3. Briefly, if $p:(M, \Pi) \rightarrow(N, \pi)$ is such a submersion, a weak splitting of it is a partition of $N=\bigsqcup_{\alpha \in A} N_{\alpha}$ into complete Poisson submanifolds and liftings $i: N_{\alpha} \rightarrow$ $M$ of the restrictions of the submersions $\left.p\right|_{p^{-1}\left(N_{\alpha}\right)}$ such that $\left(i_{\alpha}\left(N_{\alpha}\right), i_{\alpha *}\left(\left.\pi\right|_{N_{\alpha}}\right)\right)$ are Poisson-Dirac submanifolds of $(M, \Pi)$. If such a weak splitting exists, then the symplectic leaves of the base $(N, \pi)$ are just the connected components of the inverse images under the maps $i_{\alpha}$ of the symplectic leaves of $(M, \Pi)$. It is interesting to note that in the present situation the needed partition of $\left(G / P_{J}^{+}, \pi_{J}\right)$ is exactly the partition into Schubert cells. The second proof of Theorem 0.4 grew out of our attempt to understand geometrically the observation [3, Remark 3.10].

0.6. As was noted earlier, many interesting quadratic algebras are quantizations of the algebras of functions on particular Schubert cells in particular flag varieties. The idea of weak splittings of surjective Poisson submersions also suggests that the primitive ideals of those algebras can be obtained as push forwards from the primitive ideals of localizations of quotients of the quantized algebras of functions on simple groups under nonalgebra (!) maps. We plan to return to this in a forthcoming publication.

In the case when the parabolic subgroup $P_{J}^{+}$has abelian unipotent radical, there exists a real form $G_{0}$ of $G$ for which $G_{0} \cap L_{J}$ is a maximal compact subgroup of both $G_{0}$ and $L_{J}$. In [19], an explicit order-reversing bijection is constructed between the $L_{J}$-orbits on $G / P_{J}^{+}$and the $G_{0}$-orbits on $G / P_{J}^{+}$which were studied in great detail in the framework of symmetric spaces by Wolf 24, Takeuchi 23, and others. It is interesting to understand whether this bijection can be further refined to a bijection between torus orbits of symplectic leaves of the Poisson structure $\pi_{J}$ and orbits of leaves of a real Poisson structure on $G / P_{J}^{+}$, e.g. the one of Foth and $\mathrm{Lu}[8$.

Finally, let us note that parts (i) and (ii) of Theorem 0.4 show that each intersection of dual Schubert varieties in $G / P_{J}^{+}$(i.e., each Richardson variety [2] in $\left.G / P_{J}^{+}\right)$is the Zariski closure of a single $H$-orbit of symplectic leaves. This suggests the possibility to construct explicit degenerations of Richardson varieties by deforming algebraically the Poisson structure $\pi_{J}$ and looking at how torus orbits of symplectic leaves deform. This could provide a Poisson geometric approach to Schubert calculus.

0.7. We conclude the Introduction with some notation to be used later in the paper.

If $\left\{\alpha_{1}, \ldots, \alpha_{N}\right\}$ denotes the set of positive simple roots of the reductive Lie algebra $\mathfrak{g}$ and $\gamma=\sum_{i} n_{i} \alpha_{i}$ is an arbitrary root of $\mathfrak{g}$, then the $\alpha_{j}$ height of $\gamma$ is defined by $n_{\alpha_{j}}(\gamma)=n_{j}$. The support of $\gamma$ is defined by

$$
\operatorname{supp} \gamma=\left\{\alpha_{j} \mid 1 \leq j \leq N, \quad n_{\alpha_{j}}(\gamma) \neq 0\right\} .
$$

If $V$ is a vector space, then for a given $\pi \in \bigwedge^{2} V$ we will use the standard notation for the linear map

$$
\left.\pi^{\sharp}: V^{*} \rightarrow V, \quad \pi^{\sharp}(\xi)=\xi\right\rfloor \pi .
$$


For a subspace $V_{1} \subset V$, we set

$$
\left(V_{1}\right)^{0}=\left\{\xi \in V^{*} \mid \xi(v)=0 \text { for all } v \in V_{1}\right\} .
$$

In particular, this notation will be used for a Poisson bivector $\pi \in\left(M, \bigwedge^{2} T M\right)$, in which case $\pi^{\sharp}: T^{*} M \rightarrow T M$ is a bundle map. A submanifold $X$ of $(M, \pi)$ will be called a complete Poisson submanifold if it is stable under all Hamiltonian flows, that is, if it is a union of symplectic leaves.

Given an algebraic group $G$ and an element $g \in G$, we will denote by $\operatorname{Ad}_{g}$ both the conjugation action of $g$ on $G, \operatorname{Ad}_{g}(h)=g h g^{-1}$, and the adjoint action of $g$ on Lie $G$. As usual, $\operatorname{ad}_{x}$ will be used for the adjoint action of Lie $G$ on itself.

As in Part I, we will use the following convention to distinguish between double cosets and orbits in homogeneous spaces. For two subgroups $C$ and $D$ of a group $G$ and an element $g \in G:(1)$ The notation $C g D$ will denote the double coset of $g$ in $G$, and (2) the notation $C . g D$ will denote the $C$-orbit of $g D \in G / D$.

Finally, for a locally closed subvariety $Y$ of an algebraic variety $X$ and a subset $Z$ of $Y, \mathrm{Cl}_{Y}(Z)$ will denote the Zariski closure of $Z$ in $Y$.

\section{TORUS ORBITS OF SYMPLECTIC LEAVES IN FLAG VARIETIES via Poisson homogeneous spaces}

1.1. In this section we study the geometry of the Poisson structure $\pi_{J}$ on the flag variety $G / P_{J}^{+}$by the techniques of Poisson homogeneous spaces; cf. [3, Section 1].

First we introduce some more notation to be used in the rest of the paper and recall basic facts on minimal and maximal length representatives for cosets in Weyl groups. Denote the set of all roots of $\left(\mathfrak{l}_{J}, \mathfrak{h}\right)$ by $\Delta_{J}$ and the corresponding subset of positive roots by $\Delta_{J}^{+}=\Delta_{J} \cap \Delta^{+}$. Recall that the (unique) minimal length and maximal length representatives $w$ of a coset in $W / W_{J}$ are characterized respectively by

$$
w\left(\Delta_{J}^{+}\right) \subset \Delta^{+}
$$

and

$$
w\left(\Delta_{J}^{+}\right) \subset-\Delta^{+} .
$$

Recall that there are two natural bijections between $W_{\min }^{J}$ and $W_{\max }^{J}$ given by

$$
v \in W_{\min }^{J} \mapsto v w_{\circ}^{J} \in W_{\max }^{J} \quad \text { and } \quad v \in W_{\min }^{J} \mapsto w_{\circ} v \in W_{\max }^{J},
$$

where $w_{\circ}$ and $w_{\circ}^{J}$ are the longest elements of $W$ and $W_{J}$.

1.2. Recall that, in the terminology of [3, $\S 1.3-1.4]$, the standard Poisson algebraic group $\left(G, \pi_{G}\right)$ is a part of the algebraic Manin triple $\left(G \times G, G_{\text {diag }}, F\right)$, where $G_{\text {diag }}$ denotes the diagonal subgroup of $G \times G$ and $F$ is the dual Poisson algebraic group given by

$$
F=\left\{\left(h u^{+}, h^{-1} u^{-}\right) \mid h \in H, u^{ \pm} \in U^{ \pm}\right\} .
$$

On the level of Lie algebras, we have the standard Manin triple $\left(\mathfrak{g} \oplus \mathfrak{g}, \mathfrak{g}_{\text {diag }}, \mathfrak{g}^{*}\right)$, where $\mathfrak{g}^{*}=$ Lie $F$ and the bilinear form on $\mathfrak{g} \oplus \mathfrak{g}$ is

$$
\left\langle\left(x_{1}, x_{2}\right),\left(y_{1}, y_{2}\right)\right\rangle=\left\langle x_{1}, y_{1}\right\rangle-\left\langle x_{2}, y_{2}\right\rangle
$$

in terms of the nondegenerate bilinear form $\langle.,$.$\rangle on \mathfrak{g}$ fixed in $\S 0.2$. 
1.3. Proposition. (a) The orthogonal complement of $\mathfrak{p}_{J}^{+}$in the dual Lie bialgebra $\mathfrak{g}^{*}$ for the standard Lie bialgebra structure on $\mathfrak{g}$ is $\left(\mathfrak{p}_{J}^{+}\right)^{\perp}=\mathfrak{n}_{J}^{+} \oplus\{0\}$.

(b) The standard parabolic subgroup $P_{J}^{+}$of $G$ is a Poisson algebraic subgroup for the standard Poisson structure $\pi_{G}$.

(c) The pair $\left(G / P_{J}^{+}, \eta_{J *}\left(\pi_{G}\right)\right)$ is a Poisson homogeneous space for the standard Poisson algebraic group $\left(G, \pi_{G}\right)$. The Poisson structure $\eta_{J *}\left(\pi_{G}\right)$ is equal to $-\chi\left(r_{\mathfrak{g}}\right)$ and will be denoted by $\pi_{J}$ for brevity.

(d) The Drinfeld Lagrangian subalgebra (cf. [7]) of the base point $x_{1}^{J}=e P_{J}^{+}$of the Poisson homogeneous space in (c) is

$$
\overline{\mathfrak{l}}_{J}=\left\{\left(l+n_{1}^{+}, l+n_{2}^{+}\right) \mid l \in \mathfrak{l}_{J}, n_{i}^{+} \in \mathfrak{n}_{J}^{+}\right\} \subset \mathfrak{g} \oplus \mathfrak{g} \cong D(\mathfrak{g}) .
$$

It is the tangent Lie algebra of the connected algebraic subgroup

$$
\bar{L}_{J}=\left\{\left(l u_{1}^{+}, l u_{2}^{+}\right) \mid l \in L_{J}, u_{i}^{+} \in U_{J}^{+}\right\}
$$

of $G \times G$. In particular, $\left(G / P_{J}^{+}, \pi_{J}\right)$ is an algebraic Poisson homogeneous space for the standard Poisson algebraic group $\left(G, \pi_{G}\right)$ in the terminology of [3, Definition 1.7].

The proof of Proposition 1.3 is analogous to that of [3, Proposition 3.2] and will be omitted.

Below we will need the following well known Lemma.

1.4. Lemma. All $B^{-}$-orbits on the flag variety $G / P_{J}^{+}$are parametrized by $W_{\max }^{J}$ by $w \mapsto B^{-} x_{w}^{J}$. Moreover $B^{-} x_{w}^{J}$ is biregularly isomorphic to $U_{w}^{-}($recall $(0.7))$ by

$$
u \in U_{w}^{-} \mapsto u x_{w}^{J} .
$$

For completeness, we will sketch the proof of the second part of Lemma 1.4. For any $w \in W$, one has $B^{-}=U_{w}^{-}\left(B^{-} \cap \operatorname{Ad}_{w}\left(B^{+}\right)\right)$. If $w \in W_{\text {max }}^{J}$, then (1.2) implies that $\mathfrak{n}^{-} \cap \operatorname{Ad}_{w}\left(\mathfrak{n}^{-}\right) \subset \operatorname{Ad}_{w}\left(\mathfrak{n}_{J}^{-}\right)$. Since $U_{w}^{-}$and $\operatorname{Ad}_{w}\left(U_{J}^{-}\right)$are connected subgroups of $G$, it follows that $U_{w}^{-} \subset \operatorname{Ad}_{w}\left(U_{J}^{-}\right)$, which easily implies the second statement in Lemma 1.4.

Set

$$
\Omega^{J}=\left\{\left(w_{1}, w_{2}\right) \in W_{\max }^{J} \times W \mid w_{1} \leq w_{2}\right\} .
$$

1.5. Theorem. There are finitely many $H$-orbits of symplectic leaves on $\left(G / P_{J}^{+}\right.$, $\left.\pi_{J}\right)$, bijectively parametrized by $\Omega^{J}$, and all of them are smooth irreducible locally closed subvarieties of $G / P_{J}^{+}$. The $H$-orbit of leaves corresponding to $\left(w_{1}, w_{2}\right) \in \Omega^{J}$ is explicitly given by

$$
\mathcal{S}_{w_{1}, w_{2}}^{J}=\mathcal{U}_{\dot{w}_{1}, w_{2}} \cdot P_{J}^{+}
$$

(recall (0.6)) and is biregularly isomorphic to the intersection $\mathcal{B}_{w_{1}, w_{2}}=B^{-} x_{w_{1}} \cap$ $B^{+} x_{w_{2}}$ of dual Schubert cells in the full flag variety $G / B^{+}$.

In particular, the $H$-orbits of symplectic leaves on $\left(G / B^{+}, \pi\right)$ are exactly the intersections of dual Schubert cells $\mathcal{B}_{w_{1}, w_{2}}$, indexed by pairs $\left(w_{1}, w_{2}\right) \in W \times W$ such that $w_{1} \leq w_{2}$.

It is easy to see that, while $\mathcal{U}_{\dot{w}_{1}, w_{2}}$ depends on the representative of $w_{1}$ in the normalizer of $H$, the set $\mathcal{S}_{w_{1}, w_{2}}^{J}$ does not.

Proof. It follows from [3, Theorem 1.10] that the $H$-orbits of symplectic leaves in $\left(G / P_{J}^{+}, \pi_{J}\right)$ are smooth locally closed subsets of $G / P_{J}^{+}$. Moreover the same 
Theorem implies that they are exactly the irreducible components of the inverse images under the map

$$
\Delta: G / P_{J}^{+} \hookrightarrow(G \times G) / \bar{L}_{J}, \quad \Delta\left(g P_{J}^{+}\right)=(g, g) \bar{L}_{J}
$$

of the $\left(B^{+} \times B^{-}\right)$-orbits on $(G \times G) / \bar{L}_{J}$. The map $\Delta$ is an embedding because

$$
G_{\text {diag }} \cap \bar{L}_{J}=\left(P_{J}^{+}\right)_{\text {diag }} \text {. }
$$

(Recall that $G_{\text {diag }}$ denotes the diagonal subgroup of $G \times G$.)

Applying [3, Theorem 8.1], we see that each such orbit passes through the coset of $\left(\dot{w}_{2}, \dot{w}_{1}\right)$ for some $\left(w_{1}, w_{2}\right) \in W_{\min }^{J} \times W$ and that all such orbits are distinct. Because $\left(\dot{w}_{\circ}^{J}, \dot{w}_{\circ}^{J}\right) \in \bar{L}_{J}$, using (1.3) we obtain that

$$
\left\{\left(\dot{w}_{2}, \dot{w}_{1}\right) \bar{L}_{J} \mid w_{1} \in W_{\max }^{J}, w_{2} \in W\right\}
$$

is a complete, irredundant set of representatives for all $\left(B^{+} \times B^{-}\right)$-orbits on $(G \times G) / \bar{L}_{J}$. Note that

$$
\Delta^{-1}\left(\left(B^{+} \times B^{-}\right)\left(\dot{w}_{2}, \dot{w}_{1}\right) \bar{L}_{J}\right) \subseteq B^{-} x_{w_{1}}^{J} .
$$

Thus, $\Delta^{-1}\left(\left(B^{+} \times B^{-}\right)\left(\dot{w}_{2}, \dot{w}_{1}\right) \bar{L}_{J}\right)$ consists of all points $u^{-} x_{w_{1}}^{J}$, with $u^{-} \in U_{w}^{-}$, for which there exist $b^{ \pm} \in B^{ \pm}, u_{1}^{+}, u_{2}^{+} \in U_{J}^{+}$, and $l \in L_{J}$ such that

$$
u^{-} \dot{w}_{1}=b^{-} \dot{w}_{1} l u_{2}^{+}=b^{+} \dot{w}_{2} l u_{1}^{+} .
$$

From the first equality we get that $l u_{2}^{+}=\operatorname{Ad}_{\dot{w}_{1}}^{-1}\left(\left(b^{-}\right)^{-1} u^{-}\right) \in \operatorname{Ad}_{w_{1}}^{-1}\left(B^{-}\right)$. Since $w_{1} \in W_{\max }^{J}$ we have $\operatorname{Ad}_{\dot{w}_{1}}\left(u_{2}^{+}\right) \in B^{-}$; thus $l \in L_{J} \cap \operatorname{Ad}_{w_{1}}\left(B^{-}\right)$. For arbitrary $l \in L_{J} \cap \operatorname{Ad}_{w_{1}}\left(B^{-}\right)$and $u^{-} \in U_{w}^{-}$there exist $b^{-} \in B^{-}$and $u_{2}^{+} \in U_{J}^{+}$that satisfy the first equality in (1.11). Then the second equality in (1.11) implies

$$
\Delta^{-1}\left(\left(B^{+} \times B^{-}\right)\left(\dot{w}_{2}, \dot{w}_{1}\right) \bar{L}_{J}\right)=\left(U_{w_{1}}^{-} \dot{w}_{1} \cap B^{+} w_{2}\left(L_{J} \cap \operatorname{Ad}_{w_{1}}^{-1}\left(B^{-}\right)\right) U_{J}^{+}\right) \cdot P_{J}^{+} .
$$

From (1.2) one has $\operatorname{Ad}_{w_{1}}^{-1}\left(B^{-}\right)=\left(L_{J} \cap B^{+}\right)\left(U_{J}^{-} \cap \operatorname{Ad}_{w_{1}}^{-1}\left(B^{-}\right)\right)$. Thus $L_{J} \cap \operatorname{Ad}_{w_{1}}^{-1}\left(B^{-}\right)$ $=L_{J} \cap B^{+}$and

$$
\Delta^{-1}\left(\left(B^{+} \times B^{-}\right)\left(\dot{w}_{2}, \dot{w}_{1}\right) \bar{L}_{J}\right)=\left(U_{w_{1}}^{-} \dot{w}_{1} \cap B^{+} w_{2} B^{+}\right) \cdot P_{J}^{+}=\mathcal{U}_{\dot{w}_{1}, w_{2}} \cdot P_{J}^{+} .
$$

It is known that $\mathcal{U}_{\dot{w}_{1}, w_{2}}$ is nonempty if and only if $w_{2} \geq w_{1}$ (this follows from results in [12] and is proved explicitly in [6, Corollary 1.2]). On the other hand if $w_{2} \geq w_{1}$, then $\mathcal{S}_{w_{1}, w_{2}}^{J}$ is irreducible and biregularly isomorphic to $\mathcal{U}_{\dot{w}_{1}, w_{2}}$ and $\mathcal{B}_{w_{1}, w_{2}}$ because of Lemma 1.4 and [3, Theorem 2.4].

1.6. Next we describe the relation between the Poisson structures on torus orbits of leaves in different flag varieties for a fixed reductive group $G$. For a subset $J \subseteq \Gamma$, denote by

$$
\mu_{J}: G / B^{+} \rightarrow G / P_{J}^{+}
$$

the natural projection. The composition $G \stackrel{\eta}{\rightarrow} G / B^{+} \stackrel{\mu_{J}}{\longrightarrow} G / P_{J}^{+}$coincides with $\eta_{J}$ (recall (0.3)). Since the surjective maps $\eta$ and $\eta_{J}$ are Poisson, the projection $\mu_{J}$ is Poisson as well.

For all $J \subseteq \Gamma$ and $w \in W$, the sets $B^{ \pm} x_{w}^{J}$ are complete Poisson subvarieties of $\left(G / P_{J}^{+}, \pi_{J}\right)$. This follows from the facts that $\left(G / P_{J}^{+}, \pi_{J}\right)$ is a quotient of $\left(G, \pi_{G}\right)$ and $B^{ \pm}$are Poisson algebraic subgroups of $\left(G, \pi_{G}\right)$. (The statement is also a simple corollary of Theorem 1.5.) 
Proposition. For every subset $J \subseteq \Gamma$ and every $w \in W_{\max }^{J}$, the projection $\mu_{J}$ restricts to an isomorphism of Poisson varieties

$$
\left.\mu_{J}\right|_{B^{-} x_{w}}:\left(B^{-} x_{w}, \pi\right) \stackrel{\cong}{\rightrightarrows}\left(B^{-} x_{w}^{J}, \pi_{J}\right) .
$$

In particular, for $\left(w_{1}, w_{2}\right) \in \Omega^{J}$ this restricts to the Poisson isomorphism

$$
\left.\mu_{J}\right|_{\mathcal{S}_{w_{1}, w_{2}}}:\left(\mathcal{S}_{w_{1}, w_{2}}, \pi\right) \stackrel{\cong}{\cong}\left(\mathcal{S}_{w_{1}, w_{2}}^{J}, \pi_{J}\right),
$$

where for simplicity we set $\mathcal{S}_{w_{1}, w_{2}}:=\mathcal{S}_{w_{1}, w_{2}}^{\emptyset}$.

Proof. Lemma 1.4 guarantees that $\mu_{J}$ is an isomorphism between the affine spaces $B^{-} x_{w} \subset G / B^{+}$and $B^{-} x_{w}^{J} \subset G / P_{J}^{+}$. It was shown above that $B^{-} x_{w}$ and $B^{-} x_{w}^{J}$ are complete Poisson subvarieties of $\left(G / B^{+}, \pi\right)$ and $\left(G / P_{J}^{+}, \pi_{J}\right)$, respectively. The first statement now follows from the fact that $\mu_{J}$ is a Poisson mapping, and the second one is a corollary of the first.

1.7. Remark. One can first establish Proposition 1.6 and then deduce the general case of Theorem 1.5 from the case $J=\emptyset$. The proof of Theorem 1.5 in this special case is easier than the general case. This gives a somewhat simpler proof of Theorem 1.5 .

Finally we describe the Zariski closures of the torus orbits of symplectic leaves $\mathcal{S}_{w_{1}, w_{2}}^{J}$ of $\left(G / P_{J}^{+}, \pi_{J}\right)$.

1.8. Theorem. For all $\left(w_{1}, w_{2}\right) \in \Omega^{J}$, the Zariski closure of the $H$-orbit of symplectic leaves $\mathcal{S}_{w_{1}, w_{2}}^{J}$ in $\left(G / P_{J}^{+}, \pi_{J}\right)$ is equal to the union of $\mathcal{S}_{v_{1}, v_{2}}^{J}$ over those $\left(v_{1}, v_{2}\right) \in \Omega^{J}$ for which there exists $z \in W_{J}$ such that $w_{1} \leq v_{1} z$ and $w_{2} \geq v_{2} z$.

Note that in the special case of the generalized full flag variety $G / B^{+}$, Theorem 1.8 reduces to the well known fact that for the Richardson varieties,

$$
\overline{B^{-} x_{w_{1}} \cap B^{+} x_{w_{2}}}=\overline{B^{-} x_{w_{1}}} \cap \overline{B^{+} x_{w_{2}}} .
$$

The inclusion relations among the closures of the $H$-orbits $\mathcal{S}_{w_{1}, w_{2}}^{J}$ were also determined (independently) by Rietsch [20, Proposition 7.2], with different combinatorics. Moreover, Rietsch determined these relations for the nonnegative parts of the $\mathcal{S}_{w_{1}, w_{2}}^{J}$ in the sense of Lusztig [20, Theorem 6.1]. Our derivation leads to a simpler final result and finds a relation with orbit closures in wonderful group compactifications [21], [13].

Proof. By modifying Springer's Lemma 2.2 [21] or directly using [13, Lemmas 4.17 and 4.22], one gets that for all $\left(w_{1}, w_{2}\right) \in W_{\min }^{J} \times W$ inside $(G \times G) / \bar{L}_{J}$ :

$$
\begin{array}{r}
\overline{\left(B^{+} \times B^{+}\right) \cdot\left(w_{2}, w_{1}\right) \bar{L}_{J}}=\bigsqcup\left\{\left(B^{+} \times B^{+}\right) \cdot\left(v_{2}, v_{1}\right) \bar{L}_{J} \mid\left(v_{1}, v_{2}\right) \in W_{\min }^{J} \times W,\right. \\
\left.\exists z \in W_{J} \text { such that } w_{1} \geq v_{1} z, w_{2} \geq v_{2} z\right\} .
\end{array}
$$

Acting on (1.13) by $\left(e, \dot{w}_{\circ}\right)$ and using the fact that $\operatorname{Ad}_{w_{\circ}}\left(B^{+}\right)=B^{-}$and that for $w, v \in W$, we have $w \leq v$ if and only if $w_{\circ} w \geq w_{\circ} v$, we see that for $\left(w_{1}, w_{2}\right) \in$ $W_{\min }^{J} \times W$

$$
\begin{array}{r}
\overline{\left(B^{+} \times B^{-}\right) \cdot\left(w_{2}, w_{\circ} w_{1}\right) \bar{L}_{J}}=\bigsqcup\left\{\left(B^{+} \times B^{-}\right) \cdot\left(v_{2}, w_{\circ} v_{1}\right) \bar{L}_{J} \mid\left(v_{1}, v_{2}\right) \in W_{\min }^{J} \times W,\right. \\
\left.\exists z \in W_{J} \text { such that } w_{\circ} w_{1} \leq w_{\circ} v_{1} z, w_{2} \geq v_{2} z\right\} .
\end{array}
$$


We deduce from the second correspondence between $W_{\min }^{J}$ and $W_{\max }^{J}$ in (1.3) that for all $\left(w_{1}, w_{2}\right) \in W_{\max }^{J} \times W$,

$$
\begin{gathered}
\overline{\left(B^{+} \times B^{-}\right) \cdot\left(w_{2}, w_{1}\right) \bar{L}_{J}}=\bigsqcup\left\{\left(B^{+} \times B^{-}\right) \cdot\left(v_{2}, v_{1}\right) \bar{L}_{J} \mid\left(v_{1}, v_{2}\right) \in W_{\max }^{J} \times W,\right. \\
\left.\exists z \in W_{J} \text { such that } w_{1} \leq v_{1} z, w_{2} \geq v_{2} z\right\} .
\end{gathered}
$$

Now we apply [3, Lemma 2.6] for $Y$ equal to the image of the embedding $\Delta$ from (1.1) and the stratification of $(G \times G) / \bar{L}_{J}$ by $\left(B^{+} \times B^{-}\right)$-orbits. Note that $\Delta\left(G / P_{J}^{+}\right)$intersects each $\left(B^{+} \times B^{-}\right)$-orbit transversally since the diagonal of $\mathfrak{g} \oplus \mathfrak{g}$ and $\mathfrak{b}_{+} \oplus \mathfrak{b}_{-}$span $\mathfrak{g} \oplus \mathfrak{g}$. It follows that

$$
\begin{aligned}
\mathrm{Cl}_{\Delta\left(G / P_{J}^{+}\right)}\left(\Delta\left(G / P_{J}^{+}\right) \cap\left(\left(B^{+} \times B^{-}\right) \cdot\left(w_{2}, w_{1}\right) \bar{L}_{J}\right)\right) \\
\quad=\Delta\left(G / P_{J}^{+}\right) \cap \overline{\left(B^{+} \times B^{-}\right) \cdot\left(w_{2}, w_{1}\right) \bar{L}_{J} .}
\end{aligned}
$$

Recall from the proof of Theorem 1.5 that for $\left(w_{1}, w_{2}\right) \in W_{\max }^{J} \times W$,

$$
\Delta\left(G / P_{J}^{+}\right)\left(\left(B^{+} \times B^{-}\right) \cdot\left(w_{2}, w_{1}\right) \bar{L}_{J}\right) \neq \emptyset \quad \text { if and only if } w_{1} \leq w_{2},
$$

in which case

$$
\mathcal{S}_{w_{1}, w_{2}}^{J}=\Delta^{-1}\left(\left(B^{+} \times B^{-}\right) \cdot\left(w_{2}, w_{1}\right) \bar{L}_{J}\right) .
$$

The Lemma now follows from (1.14), (1.15), and the fact that $\Delta$ is an embedding.

The Zariski closures of the $H$-orbits of symplectic leaves inside each Schubert cell in $\left(G / P_{J}^{+}, \pi_{J}\right)$ have a particularly simple form.

1.9. Proposition. For each $w \in W_{\max }^{J}$ the following hold:

(i) The $H$-orbits of symplectic leaves in the Schubert cell $\left(B^{-} x_{w}^{J} \subset G / P_{J}^{+}\right.$, $\left.\left.\pi_{J}\right|_{B^{-} x_{w}^{J}}\right)$ are parametrized by $W^{\geq w}=\left\{w_{2} \in W \mid w_{2} \geq w\right\}$ by

$$
w_{2} \in W^{\geq w} \mapsto \mathcal{S}_{w, w_{2}}^{J}=\mathcal{U}_{w, w_{2}} \cdot P_{J}^{+} .
$$

(ii) For each $w_{2} \in W^{\geq w}$, the Zariski closure of $\mathcal{S}_{w, w_{2}}^{J}$ inside the Schubert cell $B^{-} x_{w}^{J}$ consists of all $\mathcal{S}_{w, v_{2}}^{J}$ for $v_{2} \in W$ such that $w \leq v_{2} \leq w_{2}$.

This proposition generalizes Theorems 3.9 and 3.13 from [3].

Proof. The first part follows from

$$
\mathcal{S}_{w, w_{2}}^{J}=\mathcal{U}_{w, w_{2}} \cdot P_{J}^{+} \subset\left(U_{w}^{-} w\right) \cdot P_{J}^{+}=B^{-} x_{w}^{J}
$$

and the fact that the Schubert cells $B^{-} x_{w}^{J}$ for $w \in W_{\max }^{J}$ partition $G / P_{J}^{+}$.

It is easy to deduce the second part from Theorem 1.8, but we offer a direct proof which better explains the result. by

In terms of the isomorphism $\left.\mu_{J}\right|_{B^{-} x_{w}}: B^{-} x_{w} \rightarrow B^{-} x_{w}^{J}$ (cf. $\left.\S 1.6\right), \mathcal{S}_{w, w_{2}}^{J}$ is given

$$
\mathcal{S}_{w, w_{2}}^{J}=\left(\left.\mu_{J}\right|_{B^{-} x_{w}}\right)^{-1}\left(B^{-} x_{w} \cap B^{+} x_{w_{2}}\right) .
$$

Applying [3, Lemma 2.6] for the partition $X=G / B^{+}=\bigsqcup_{w_{1} \in W} B^{+} x_{w_{1}}$ and $Y=$ $B^{-} x_{w}$, and using the standard formulas for closures of Schubert cells, leads to

$$
\mathrm{Cl}_{B^{-} x_{w}}\left(B^{-} x_{w} \cap B^{+} x_{w_{2}}\right)=\bigsqcup_{v_{2} \in W, w \leq v_{2} \leq w_{2}}\left(B^{-} x_{w} \cap B^{+} x_{v_{2}}\right) .
$$


The second part of the Proposition follows from this, applying once again the isomorphism $\left.\mu_{J}\right|_{B^{-} x_{w}}$.

\section{Weak splittings of surjective Poisson submersions}

2.1. First we recall the definition of a Poisson-Dirac submanifold of a Poisson manifold, given by Crainic and Fernandes in [5, Section 9].

Definition. Assume that $(M, \Pi)$ is a smooth (real or complex) Poisson manifold. A submanifold $X$ of $M$ is called a Poisson-Dirac submanifold if the following two conditions are satisfied:

(i) For each symplectic leaf $S$ of $(M, \Pi)$, the intersection $S \cap X$ is clean (i.e., it is smooth and $T_{x}(S \cap X)=T_{x} S \cap T_{x} X$ for all $\left.x \in S \cap X\right)$ and $S \cap X$ is a symplectic submanifold of $\left(S,\left(\left.\Pi\right|_{S}\right)^{-1}\right)$.

(ii) The family of symplectic structures $\left.\left(\left.\Pi\right|_{S}\right)^{-1}\right|_{S \cap X}$ is induced by a smooth Poisson structure $\pi$ on $X$.

Here and below, for a nondegenerate Poisson structure $\pi_{0}$ we denote by $\left(\pi_{0}\right)^{-1}$ the corresponding symplectic form.

In the setting of the above Definition, the symplectic leaves of $(X, \pi)$ are exactly the connected components of the intersections of the symplectic leaves of $(M, \Pi)$ with $X$.

2.2. The following simple criterion was proved in $[5]$.

Proposition. Assume that $(M, \Pi)$ is a Poisson manifold and that $X$ is a submanifold for which there exists a subbundle $E$ of $T_{X} M$ such that

(i) $T_{X} M=T X \oplus E$ and

(ii) the restriction of the Poisson tensor $\Pi$ to $X$ splits as

$$
\left.\Pi\right|_{X}=\pi+\pi_{E}
$$

for some smooth bivector fields $\pi \in \Gamma\left(X, \bigwedge^{2} T X\right)$ and $\pi_{E} \in \Gamma\left(X, \bigwedge^{2} E\right)$.

Then $X$ is a Poisson-Dirac submanifold of $(M, \Pi)$, and the induced Poisson structure on it coincides with $\pi$.

Crainic and Fernandes call submanifolds satisfying the conditions of Proposition 2.2 Poisson-Dirac submanifolds admitting a Dirac projection. Earlier, Xu [26] investigated such submanifolds with an extra property, namely that $E^{0}$ is a Lie subalgebroid of $T^{*} M$, equipped with the standard cotangent bundle algebroid structure (recall (0.9)).

2.3. Definition. Assume that $(M, \Pi)$ and $(N, \pi)$ are Poisson manifolds and that $p:(M, \Pi) \rightarrow(N, \pi)$ is a surjective Poisson submersion. A weak splitting of $p$ is a partition

$$
N=\bigsqcup_{\alpha \in A} N_{\alpha}
$$

of $(N, \pi)$ into complete Poisson submanifolds such that for each $\alpha \in A$ there exists a smooth lifting $i_{\alpha}: N_{\alpha} \rightarrow M$ (of $\left.p\right|_{p^{-1}\left(N_{\alpha}\right)}: p^{-1}\left(N_{\alpha}\right) \rightarrow N_{\alpha}$ ) with the properties:

(i) $i_{\alpha}\left(N_{\alpha}\right)$ is a Poisson-Dirac submanifold of $(M, \Pi)$ and

(ii) the induced Poisson structure on $i_{\alpha}\left(N_{\alpha}\right)$ is $i_{\alpha *}\left(\left.\pi\right|_{N_{\alpha}}\right)$.

Note that $i_{\alpha}$ is not required to be a Poisson map. An important special case is illustrated in algebraic terms in Proposition 2.6. 
2.4. Remark. If a surjective Poisson submersion $p:(M, \Pi) \rightarrow(N, \pi)$ admits a weak splitting as in Definition 2.3, then the symplectic foliation of $(N, \pi)$ is easily described in terms of the symplectic foliation of $(M, \Pi)$. Namely, each symplectic leaf of $(N, \pi)$ lies entirely in one of the submanifolds $N_{\alpha}$ and is of the type $i_{\alpha}^{-1}\left(S \cap i_{\alpha}\left(N_{\alpha}\right)\right)^{\circ}$, where $S$ is a symplectic leaf of $M$ and $\left(S \cap i_{\alpha}\left(N_{\alpha}\right)\right)^{\circ}$ is a connected component of $\left(S \cap i_{\alpha}\left(N_{\alpha}\right)\right)$.

2.5. The following Proposition provides a sufficient condition for the condition (ii) in Definition 2.3 which is easier to check.

Proposition. Assume that $p:(M, \Pi) \rightarrow(N, \pi)$ is a surjective Poisson submersion. Let

$$
N=\bigsqcup_{\alpha \in A} N_{\alpha}
$$

be a partition of $(N, \pi)$ into complete Poisson submanifolds such that for each $\alpha \in$ $A$, there exists a smooth lifting $i_{\alpha}: N_{\alpha} \rightarrow M\left(\right.$ of $\left.\left.p\right|_{p^{-1}\left(N_{\alpha}\right)}: p^{-1}\left(N_{\alpha}\right) \rightarrow N_{\alpha}\right)$ whose image is a Poisson-Dirac submanifold admitting a Dirac projection with respect to a subbundle $E_{\alpha}$ of $T_{i_{\alpha}\left(N_{\alpha}\right)} M$, cf. Proposition 2.2. If the $E_{\alpha}$ are tangent to the fibers of $p$ (i.e., the $E_{\alpha}$ contain the tangent spaces to the fibers of $p$ ), then condition (ii) in Definition 2.3 is satisfied and the families $\left\{N_{\alpha}\right\}_{\alpha \in A},\left\{i_{\alpha}\right\}_{\alpha \in A}$ provide a weak splitting of $p$.

Proof. For $m \in i_{\alpha}\left(N_{\alpha}\right)$ denote the fiber of $p$ through $m$ by $F_{m}=p^{-1}(p(m))$. Since $p:(M, \Pi) \rightarrow(N, \pi)$ is Poisson and $i_{\alpha}$ is a lifting of $\left.p\right|_{p^{-1}\left(N_{\alpha}\right)}: p^{-1}\left(N_{\alpha}\right) \rightarrow N_{\alpha}$, we have

$$
T_{m} M=T_{m}\left(i_{\alpha}\left(N_{\alpha}\right)\right) \oplus T_{m} F_{m}
$$

and

$$
\Pi_{m}-i_{\alpha *}\left(\left.\pi\right|_{N_{\alpha}}\right)_{m} \in T_{m} F_{m} \wedge T_{m} M .
$$

On the other hand, the fact that $i_{\alpha}\left(N_{\alpha}\right) \subset M$ satisfies the conditions of Proposition 2.2 implies

$$
\begin{aligned}
& \left.\Pi\right|_{i_{\alpha}\left(N_{\alpha}\right)}=\pi_{\alpha}+\pi_{E_{\alpha}}, \\
& \quad \text { for some } \pi_{\alpha} \in \Gamma\left(i_{\alpha}\left(N_{\alpha}\right), \bigwedge^{2} T\left(i_{\alpha}\left(N_{\alpha}\right)\right)\right), \pi_{E_{\alpha}} \in \Gamma\left(i_{\alpha}\left(N_{\alpha}\right), \bigwedge^{2} E_{\alpha}\right) .
\end{aligned}
$$

Putting together (2.2)-(2.4) and $T_{m} F_{m} \subseteq E_{\alpha}(m)$ gives $\pi_{\alpha}=i_{\alpha *}\left(\left.\pi\right|_{N_{\alpha}}\right)$, which is exactly condition (ii) in Definition 2.4 (taking into account Proposition 2.2).

2.6. If $p:(M, \Pi) \rightarrow(N, \pi)$ is a Poisson map, then the pull back $p^{*}:\left(C^{\infty}(N),\{., .\}_{\pi}\right)$ $\rightarrow\left(C^{\infty}(M),\{., .\}_{\Pi}\right)$ is a homomorphism of Poisson algebras and turns $C^{\infty}(M)$ into a module for the Poisson algebra $\left(C^{\infty}(N),\{., .\}_{\pi}\right)$. The following Proposition provides an algebraic characterization of an important special case of weak splittings of surjective Poisson submersions. Its proof is simple and will be left to the reader.

Proposition. Assume that $p:(M, \Pi) \rightarrow(N, \pi)$ is a surjective Poisson submersion and $i: N \rightarrow M$ a smooth lifting of $p$. Denote by Tp the subbundle of TM whose fibers are the tangent spaces to the fibers of $p$. Then the trivial partition of $N$ with one stratum and the map $i: N \rightarrow M$ provide a weak splitting of $p$ such that $\left.\Pi\right|_{i(N)} \in$ $\left.\bigwedge^{2} T i(N) \oplus \bigwedge^{2} T p\right|_{i(N)}$ if and only if $i^{*}:\left(C^{\infty}(M),\{.,\}_{\Pi}\right) \rightarrow\left(C^{\infty}(N),\{., .\}_{\pi}\right)$ is a morphism of $\left(C^{\infty}(N),\{., .\}_{\pi}\right)$ modules. 
2.7. All weak splittings of surjective Poisson submersions considered in this paper will be in the category of (complex) quasiprojective Poisson varieties. This means that in the setting of Definition 2.1, we require $X$ to be a (smooth) locally closed subvariety of the smooth quasiprojective Poisson variety $M$. In Proposition 2.2, we require $E$ to be an algebraic subbundle of $T_{X} M$. Finally, in the algebraic setting, in Definition 2.3 we require (2.1) to be an algebrogeometric stratification of $M$ (in the sense of $[3, \S 0.8])$ and the maps $i_{\alpha}$ to be algebraic.

\section{Weak splittings of surJective Poisson submersions FOR FLAG VARIETIES}

3.1. Since the Poisson structure $\pi_{G}$ vanishes on the maximal torus $H$ of $G$, the left and right regular actions of $H$ on $G$ preserve it, and thus $\pi_{G}$ descends to a Poisson structure on $G / H$. One can see this in another way: $H$ is a Poisson algebraic subgroup of $\left(G, \pi_{G}\right)$; thus $\pi_{G}$ descends to a Poisson structure on the homogeneous space $G / H$ because of [3, Theorem 1.8]. Denote the standard projection by

$$
\tau: G \rightarrow G / H, \text { and set } \pi_{G / H}=\tau_{*}\left(\pi_{G}\right) .
$$

It is clear that the projections

$$
\nu_{J}:\left(G / H, \pi_{G / H}\right) \rightarrow\left(G / P_{J}^{+}, \pi_{J}\right), \quad \nu_{J}(g H)=g P_{J}^{+}
$$

are surjective Poisson submersions. For brevity, set $\nu=\nu_{\emptyset}$. Finally, for $w \in W$, set

$$
y_{w}=w H \in G / H \text {. }
$$

The following Theorem contains the main result in this section. Based on it, a second proof of Theorem 1.5 is given in $\S 3.10$.

3.2. Theorem. Assume that $G$ is an arbitrary complex reductive algebraic group and $J$ is a subset of the set of positive simple roots $\Gamma$. The partition into Schubert cells

$$
G / P_{J}^{+}=\bigsqcup_{w \in W_{\max }^{J}} B^{-} x_{w}
$$

and the morphisms

$$
i_{w}^{J}: B^{-} x_{w}^{J} \rightarrow G / H, \quad \text { given by } i_{w}^{J}\left(u^{-} x_{w}^{J}\right) \rightarrow u^{-} y_{w}, \text { for } u^{-} \in U_{w}^{-},
$$

provide a weak splitting, in the sense of Definition 2.3, of the surjective Poisson submersion $\nu_{J}:\left(G / H, \pi_{G / H}\right) \rightarrow\left(G / P_{J}^{+}, \pi_{J}\right)$ (recall (0.5), (0.7), and (3.1)-(3.3)).

Theorem 3.2 has the following important Corollary.

3.3. Corollary. Each symplectic leaf of $\left(G / P_{J}^{+}, \pi_{J}\right)$ is a symplectic submanifold of a symplectic leaf of $\left(G / H, \pi_{G / H}\right)$. More precisely, the symplectic submanifold is precisely the image of the symplectic leaf under the corresponding morphism $i_{w}^{J}$.

The proof of Theorem 3.2 will be split into several lemmas.

3.4. We will use the identification of vector spaces

$$
T_{e} G \oplus T_{e}^{*} G=\mathfrak{g} \oplus \mathfrak{g}^{*} \cong D(\mathfrak{g}) \cong \mathfrak{g} \oplus \mathfrak{g}=T_{e} G \oplus T_{e} G
$$

coming from the embeddings of $\mathfrak{g}$ and $\mathfrak{g}^{*}$ in the double $D(\mathfrak{g}) \cong \mathfrak{g} \oplus \mathfrak{g}$ of the Lie bialgebra $\mathfrak{g}$; cf. $\$ 1.2$. This induces the identifications

$$
T_{g} G \oplus T_{g}^{*} G \cong R_{g}\left(\mathfrak{g} \oplus \mathfrak{g}^{*}\right) \cong R_{g}(\mathfrak{g} \oplus \mathfrak{g})=T_{g} G \oplus T_{g} G, \quad \text { for } g \in G .
$$


Here and below, $L_{g}$ and $R_{g}$ refer to the left and right translations $a \mapsto g a$ and $a \mapsto a g$, for $a \in G$. For simplicity of the notation, we denote in the same way the induced tangent maps $T_{a} \rightarrow T_{g a}$ and $T_{a} \rightarrow T_{a g}$. In the identifications (3.6), the tangent and cotangent spaces at $g$ correspond respectively to

$$
\begin{aligned}
T_{g} G \cong R_{g}\left(\mathfrak{g}_{\text {diag }}\right), \\
T_{g}^{*} G \cong R_{g}(\operatorname{Lie} F)=R_{g}\left\{\left(h+n^{+},-h+n^{-}\right) \mid h \in \mathfrak{h}, n^{ \pm} \in \mathfrak{n}^{ \pm}\right\} ;
\end{aligned}
$$

recall the notation in $\$ 1.2$. The pairing between them is given by (1.5).

The main reason for using the identifications (3.6) is that the graph of $\pi_{G, g}^{\sharp}$ : $T_{g}^{*} G \rightarrow T_{g} G$ (cf. (0.8)) under those identifications corresponds to the Drinfeld Lagrangian subalgebra [7] $\mathfrak{l}_{g}$ of $D(\mathfrak{g})$ for the base point $g$ of $\left(G, \pi_{G}\right)$, considered as a homogeneous space over itself. Since $\pi_{G}$ vanishes at $e$, one has $\mathfrak{l}_{e}=\operatorname{Lie} F$. Moreover, because the map $g \mapsto \mathfrak{l}_{g} \subset D(\mathfrak{g})$ is $G$-equivariant with respect to the adjoint action of $G$ on $D(\mathfrak{g}) \cong \mathfrak{g} \oplus \mathfrak{g}$ (see [7]), one has

$$
\mathfrak{l}_{g}=\operatorname{Ad}_{g}(\operatorname{Lie} F)=\operatorname{Ad}_{g}\left(\left\{\left(h+n^{+},-h+n^{-}\right) \mid h \in \mathfrak{h}, n^{ \pm} \in \mathfrak{n}^{ \pm}\right\}\right) .
$$

Taking into account $R_{g} \circ \mathrm{Ad}_{g}=L_{g}$, this leads us to the following result.

3.5. Lemma. In the identifications (3.6), the graph of $\pi_{G, g}^{\sharp}: T_{g}^{*} G \rightarrow T_{g} G$ (cf. (0.8)), corresponds to the subspace

$$
R_{g}\left(\operatorname{Ad}_{g} F\right)=L_{g}(\operatorname{Lie} F)=L_{g}\left(\left\{\left(h+n^{+},-h+n^{-}\right) \mid h \in \mathfrak{h}, n^{ \pm} \in \mathfrak{n}^{ \pm}\right\}\right) \subset T_{g} G \oplus T_{g} G .
$$

We will further need the following well known result.

3.6. Lemma. If $V_{1}$ and $V_{2}$ are subspaces of a finite dimensional vector space $V$ and $\pi \in \bigwedge^{2} V$, then

$$
\pi^{\sharp}\left(V_{1}^{0}\right) \subseteq V_{2} \quad \Longleftrightarrow \quad \pi \in \bigwedge^{2} V_{1}+\bigwedge^{2} V_{2} .
$$

Sketch of the proof. The standard nondegenerate pairing between $V \oplus V$ and $V^{*} \oplus$ $V^{*}$ restricts to a nondegenerate pairing between $\bigwedge^{2} V$ and $\bigwedge^{2} V^{*}$. Then $\pi^{\sharp}\left(V_{1}^{0}\right) \subseteq$ $V_{2}$ if and only if $\pi \in\left(V_{1}^{0} \wedge V_{2}\right)^{0}$. One easily checks that for all subspaces $V_{1}$ and $V_{2}$ of $V$, one has $\left(V_{1}^{0} \wedge V_{2}\right)^{0}=\bigwedge^{2} V_{1}+\bigwedge^{2} V_{2}$.

3.7. For all $w \in W$, define the following algebraic subbundles $\widetilde{E}_{w}$ and $E_{w}$ of $T_{H U_{w}^{-} w} G$ and $T_{U_{w}^{-} y_{w}} G / H$, respectively:

$$
\begin{aligned}
\widetilde{E}_{w}(b w) & =L_{b w}\left(\mathfrak{b}^{+}\right)+R_{b w}\left(\mathfrak{n}^{+} \cap \operatorname{Ad}_{w}\left(\mathfrak{n}^{-}\right)\right) \subset T_{b w}(G), \quad \text { for } b \in H U_{w}^{-}, \\
E_{w} & =\tau_{*}\left(\widetilde{E}_{w}\right) \subset T_{U_{w}^{-} y_{w}} G / H
\end{aligned}
$$

recall (3.4). It is easy to see that the push-forward in (3.8) does not depend on the choice of preimage. For a subvariety $N \subset G$ such that $N=\tau^{-1} \tau(N)$, denote by $T\left(\left.\tau\right|_{N}\right)$ the bundle over $N$ whose fibers are the tangent spaces to the fibers of $\left.\tau\right|_{N}$ :

$$
T\left(\left.\tau\right|_{N}\right)(g)=L_{g}(\mathfrak{h}) \subset T_{g} G, \quad g \in N .
$$

The fact that the $E_{w}$ are algebraic bundles follows from $\widetilde{E}_{w} \supset T\left(\left.\tau\right|_{H U_{w}^{-} w}\right)$ : for all $w \in W$ and $b \in H U_{w}^{-}$, one has $\widetilde{E}_{w}(b w) \supset L_{b w}\left(\mathfrak{b}^{+}\right) \supset L_{b w}(\mathfrak{h})=T\left(\left.\tau\right|_{H U_{w}^{-}}\right)(b w)$. 
Proposition. For every $w \in W$, the following hold:

(1) $T\left(H U_{w}^{-} w\right)+\widetilde{E}_{w}=T_{H U_{w}^{-} w} G$, $T\left(H U_{w}^{-} w\right) \cap \widetilde{E}_{w}=T\left(\left.\tau\right|_{H U_{w}^{-} w}\right)$, $\left.\pi_{G}\right|_{H U_{w}^{-} w} \in \bigwedge^{2} T\left(H U_{w}^{-} w\right)+\bigwedge^{2} \widetilde{E}_{w}$, and

(2) $T\left(U_{w}^{-} y_{w}\right) \oplus E_{w}=T_{U_{w}^{-} y_{w}} G$, $\left.\pi_{G / H}\right|_{U_{w}^{-} y_{w}} \in \bigwedge^{2} T\left(U_{w}^{-} y_{w}\right) \oplus \bigwedge^{2} E_{w}$ recall (0.4), (3.3), (3.4), (3.8), and (3.9).

Proof. Part (2) follows from part (1). The following inclusions imply the first statement in part (1):

$$
\begin{aligned}
T_{b w}\left(H U_{w}^{-} w\right)+\widetilde{E}_{w}(u w) & \supset R_{b w}\left(\mathfrak{b}^{-} \cap \operatorname{Ad}_{w}\left(\mathfrak{b}^{-}\right)+\mathfrak{n}^{+} \cap \operatorname{Ad}_{w}\left(\mathfrak{n}^{-}\right)\right)+L_{b w}\left(\mathfrak{b}^{+}\right) \\
& =R_{b w}\left(\operatorname{Ad}_{w}\left(\mathfrak{b}^{-}\right)\right)+L_{u w}\left(\mathfrak{b}^{+}\right)=R_{b w}\left(\operatorname{Ad}_{b w}\left(\mathfrak{b}^{-}\right)\right)+L_{b w}\left(\mathfrak{b}^{+}\right) \\
& =L_{b w}\left(\mathfrak{b}^{-}+\mathfrak{b}^{+}\right)=T_{b w}(G),
\end{aligned}
$$

where $w \in W$ and $b \in U_{w}^{-}$. We used the fact that $b \in H U_{w}^{-} \subset \operatorname{Ad}_{w}\left(B^{-}\right)$and thus $\operatorname{Ad}_{b w}\left(\mathfrak{b}^{-}\right)=\operatorname{Ad}_{w}\left(\mathfrak{b}^{-}\right)$.

It is clear that

$$
T\left(H U_{w}^{-} w\right) \cap \widetilde{E}_{w} \supset T\left(\left.\tau\right|_{H U_{w}^{-} w}\right)
$$

because $H U_{w}^{-} w=U_{w}^{-} w H$. Next we show the opposite inclusion. Fix $b \in H U_{w}^{-}$. If $x \in \mathfrak{g}$ and $R_{b w}(x) \in T_{b w}\left(H U_{w}^{-} w\right) \cap \widetilde{E}_{w}$, then $x=x_{1}=\operatorname{Ad}_{b w}(y)+x_{2}$ for some $y \in \mathfrak{b}^{+}$and $x_{1} \in \mathfrak{b}^{-} \cap \operatorname{Ad}_{w}\left(\mathfrak{b}^{-}\right)$, and $x_{2} \in \mathfrak{n}^{+} \cap \operatorname{Ad}_{w}\left(\mathfrak{n}^{-}\right)$. So

$$
\operatorname{Ad}_{b w}(y)=x_{1}-x_{2} \in \mathfrak{b}^{-} \cap \operatorname{Ad}_{w}\left(\mathfrak{b}^{-}\right)+\mathfrak{n}^{+} \cap \operatorname{Ad}_{w}\left(\mathfrak{n}^{-}\right)=\operatorname{Ad}_{w}\left(\mathfrak{b}^{-}\right)=\operatorname{Ad}_{b w}\left(\mathfrak{b}^{-}\right) .
$$

Taking into account $\operatorname{Ad}_{b w}(y) \in \operatorname{Ad}_{b w}\left(\mathfrak{b}^{+}\right)$, one obtains $y \in h$ and $x_{2}=0$, and consequently $x \in \mathfrak{h}$. This proves the opposite inclusion to (3.10) and completes the proof of the second statement in (1).

In the remainder of this proof we show the third property in part (1). Under the identifications (3.6), the tangent space $T_{b w}\left(H U_{w}^{-} w\right)$ corresponds to

$$
R_{b w}\left(\left(\mathfrak{b}^{-} \cap \operatorname{Ad}_{w}\left(\mathfrak{b}^{-}\right)\right)_{\text {diag }}\right) \quad \text { for all } b \in H U_{w}^{-} .
$$

Recall that the images of the tangent and the cotangent spaces to $G$ in (3.6) are given by (3.7) and the pairing between them is given by (1.5); see $\S 3.4$. By a direct computation, one checks that under the identifications (3.6), $\left(T_{b w}\left(H U_{w}^{-} w\right)\right)^{0}$ corresponds to $R_{b w}\left(\left(\mathfrak{n}^{+} \cap \operatorname{Ad}_{w}\left(\mathfrak{n}^{-}\right)\right) \oplus \mathfrak{n}^{-}\right)$; see (0.8)-(0.9). (Here and below, for two subalgebras $\mathfrak{f}_{1}, \mathfrak{f}_{2} \subseteq \mathfrak{g}$, by $\mathfrak{f}_{1} \oplus \mathfrak{f}_{2}$ we denote the canonical direct sum subalgebra of $\mathfrak{g} \oplus \mathfrak{g}$ and not the possible direct sum inside $\mathfrak{g}$.) Recall that the graph of $\pi_{G}^{\sharp}$ : $T_{b w}^{*} G \rightarrow T_{b w} G$ is given by Lemma 3.5. If $R_{b w}(x) \in \pi_{G}^{\sharp}\left(\left(T_{b w}\left(H U_{w}^{-} w\right)\right)^{0}\right)$, then there exist $n^{+} \in \mathfrak{n}^{+} \cap \operatorname{Ad}_{w}\left(\mathfrak{n}^{-}\right)$and $n^{-} \in \mathfrak{n}^{-}$such that

$$
\left(x+n^{+}, x+n^{-}\right) \in \operatorname{Ad}_{b w}(\operatorname{Lie} F) \subset \operatorname{Ad}_{b w}\left(\mathfrak{b}^{+} \oplus \mathfrak{b}^{-}\right) .
$$

Comparing the first components gives $x \in \operatorname{Ad}_{b w}\left(\mathfrak{b}^{+}\right)+\mathfrak{n}^{+} \cap \operatorname{Ad}_{w}\left(\mathfrak{n}^{-}\right)$, and consequently

$$
\begin{aligned}
\pi_{G}^{\sharp}\left(\left(T_{b w}\left(H U_{w}^{-} w\right)\right)^{0}\right) & \subset R_{u w}\left(\operatorname{Ad}_{b w}\left(\mathfrak{b}^{+}\right)+\mathfrak{n}^{+} \cap \operatorname{Ad}_{w}\left(\mathfrak{n}^{-}\right)\right) \\
& =L_{b w}\left(\mathfrak{b}^{+}\right)+R_{b w}\left(\mathfrak{n}^{+} \cap \operatorname{Ad}_{w}\left(\mathfrak{n}^{-}\right)\right)=\widetilde{E}_{w}(b w) .
\end{aligned}
$$

Now the third statement in part (1) follows from Lemma 3.6. 
3.8. Proof of Theorem 3.2. First we prove the Theorem in the case $J=\emptyset$. For all $w \in W$, the tangent spaces to the fibers of $\nu_{\emptyset}$ inside $U_{w}^{-} y_{w}$ are $\eta_{*}\left(L_{b w}\left(\mathfrak{b}^{+}\right)\right)$ for $b \in H U_{w}^{-}$, and they are contained in $E_{w}$. Proposition 2.5 and part (2) of Proposition 3.7 imply that

$$
\left.\pi_{G / H}\right|_{U_{w}^{-} y_{w}}-i_{w *}^{\emptyset}\left(\left.\pi\right|_{B^{-} x_{w}}\right) \in \bigwedge^{2} E_{w}, \quad \text { for all } w \in W,
$$

which establishes the Theorem in the case $J=\emptyset$.

For the general case, in addition to Proposition 3.7 (2), we need to prove that

$$
\left.\pi_{G / H}\right|_{U_{w}^{-} y_{w}}-i_{w *}^{J}\left(\left.\pi_{J}\right|_{B^{-} x_{w}^{J}}\right) \in \bigwedge^{2} E_{w}, \quad \text { for all } J \subset \Gamma, w \in W^{J} .
$$

Because $i_{w}^{J}=i_{w}^{\emptyset} \circ\left(\left.\mu_{J}\right|_{B^{-} x_{w}}\right)^{-1}$ and

$$
\left.\mu_{J}\right|_{B^{-} x_{w}}:\left(B^{-} x_{w},\left.\pi\right|_{B^{-} x_{w}}\right) \rightarrow\left(B^{-} x_{w}^{J},\left.\pi_{J}\right|_{B^{-} x_{w}^{J}}\right)
$$

is a Poisson isomorphism for all $w \in W^{J}$ (recall (1.12) and Proposition 1.6), we get that

$$
i_{w *}^{J}\left(\left.\pi_{J}\right|_{B^{-} x_{w}^{J}}\right)=i_{w *}^{\emptyset}\left(\left.\pi\right|_{B^{-} x_{w}}\right), \quad \text { for all } w \in W^{J} .
$$

Equations (3.11) and (3.13) imply (3.12), and this completes the proof of the Theorem.

3.9. Lemma. The $H$-orbits of symplectic leaves of $\left(G / H, \pi_{G / H}\right)$ are exactly the projections $\tau\left(B^{-} w_{1} B^{-} \cap B^{+} w_{2} B^{+}\right)$of the double Bruhat cells of $G$ onto $G / H$, for $w_{1}, w_{2} \in W$.

Sketch of the proof. The proof of the Lemma is analogous to the well known fact that the $H$-orbits of symplectic leaves of $\left(G, \pi_{G}\right)$ are the double Bruhat cells of $G$. The Drinfeld Lagrangian subalgebra of the base point $e H$ of $G / H$ is $\operatorname{Lie}\left(H_{\text {diag }}\left(U^{+} \times U^{-}\right)\right)$; see [3, Theorem 1.8]. The $H$-orbits of symplectic leaves of $\left(G / H, \pi_{G / H}\right)$ are the inverse images of the $\left(B^{+} \times B^{-}\right)$-orbits on $(G \times G) / H_{\text {diag }}\left(U^{+} \times U^{-}\right)$under the map

$$
G / H \rightarrow(G \times G) / H_{\text {diag }}\left(U^{+} \times U^{-}\right), \quad g H \mapsto(g, g) H_{\text {diag }}\left(U^{+} \times U^{-}\right) .
$$

By the Bruhat Lemma, the $\left(B^{+} \times B^{-}\right)$-orbits on $(G \times G) / H_{\text {diag }}\left(U^{+} \times U^{-}\right)$are parametrized by $W \times W$ via

$$
W \times W \ni\left(w_{1}, w_{2}\right) \longmapsto\left(B^{+} \times B^{-}\right)\left(w_{2}, w_{1}\right) H_{\text {diag }}\left(U^{+} \times U^{-}\right) .
$$

Finally, the inverse images of the above orbits under (3.14) are exactly the projections $\tau\left(B^{-} w_{1} B^{-} \cap B^{+} w_{2} B^{+}\right)$.

3.10. A second proof of Theorem 1.8. For $w \in W^{J}$ and $w_{1}, w_{2} \in W$, the intersection of $\tau\left(B^{-} w_{1} B^{-} \cap B^{+} w_{2} B^{+}\right)$with $i_{w}^{J}\left(B^{-} x_{w}^{J}\right)=U_{w}^{-} y_{w}$ is nonempty only if $w_{1}=w$ and $w_{2} \geq w$, because it lies inside $\tau\left(B^{-} w \cap B^{-} w_{1} B^{-}\right)$(thus $w_{1}=w$ ) and consequently inside $B^{-} w B^{+} \cap B^{+} w_{2} B^{+}$(thus $w_{2} \geq w$ by [6, Corollary 1.2]). If $w_{1}=w$ and $w_{2} \geq w$, then the intersection of $\tau\left(B^{-} w_{1} B^{-} \cap B^{+} w_{2} B^{+}\right)$with the image of $i_{w}^{J}$ is $\tau\left(U_{\dot{w}, w_{2}}\right)$ (cf. (0.6)) and is a nonempty irreducible subvariety of $G / H$ by [3, Theorem 2.3]. Theorem 3.2, Lemma 3.9, and the argument of the proof of [3, Theorem 1.8] imply that for each $w_{2} \in W$ with $w_{2} \geq w$, the set $\tau\left(\mathcal{U}_{\dot{w}, w_{2}}\right)$ is a (single) $H$-orbit of symplectic leaves of $\left(i_{w}^{J}\left(B^{-} x_{w}^{J}\right), i_{w *}^{J}\left(\left.\pi_{J}\right|_{B^{-} x_{w}^{J}}\right)\right)$. Thus, the 
$H$-orbits of symplectic leaves of $\left(B^{-} x_{w}^{J},\left.\pi_{J}\right|_{B^{-} x_{w}^{J}}\right)$ are

$$
\left(i_{w}^{J}\right)^{-1} \circ \tau\left(\mathcal{U}_{\dot{w}, w_{2}}\right)=\mathcal{U}_{\dot{w}, w_{2}} . P_{J}^{+}=\mathcal{S}_{w, w_{2}}^{J}, \quad w_{2} \in W, w_{2} \geq w .
$$

This completes the second proof of the Theorem.

Let us note that although in the second proof of Theorem 1.8 we still used the theory of Poisson homogeneous spaces in Lemma 3.9, we avoided the combinatorics arguments from the first proof. The latter were replaced by the geometric construction of weak splittings of Poisson submersions. In addition, in a subsequent publication we will demonstrate that those geometric arguments can be extended to the quantum situation, while we are not aware of any quantum version of the dressing orbit method that provides a classification of $H$-invariant prime ideals of some class of associative algebras.

\section{HeRmitian SYMmetriC SPACES OF COMPACT TYPE}

4.1. In this section we investigate the Poisson structure $\pi_{J}$ on $G / P_{J}^{+}$for the case when the unipotent radical $U_{J}^{+}$of $P_{J}^{+}$is abelian and $G$ is a simple algebraic group. The flag varieties $G / P_{J}^{+}$of this type exhaust all irreducible Hermitian symmetric spaces of compact type, 24. We show that in this case all $L_{J}$-orbits on $G / P_{J}^{+}$are complete Poisson subvarieties with respect to the Poisson structure $\pi_{J}$. We then use the results of Richardson, Röhrle, and Steinberg [19] on special representatives for the $L_{J}$-orbits on $G / P_{J}^{+}$. We prove that the Poisson structure $\pi_{J}$ vanishes at all such special base points, and as a result of this $\left(G / P_{J}^{+}, \pi_{J}\right)$ stratifies into complete Poisson subvarieties, each of which is a quotient of $L_{J}$, equipped with the standard Poisson structure.

First, set

$$
r_{J}=\sum_{\alpha \in \Delta_{J}^{+}} \frac{\langle\alpha, \alpha\rangle}{2} e_{\alpha} \wedge f_{\alpha} \quad \text { and } \quad \pi_{L_{J}}=L\left(r_{J}\right)-R\left(r_{J}\right) ;
$$

cf. (0.1) and (0.2). These are respectively the standard $r$-matrix and the standard Poisson structure on the reductive group $L_{J}$. It is well known that $\left(L_{J}, \pi_{J}\right)$ is a Poisson algebraic subgroup of $\left(G, \pi_{G}\right)$. Also set

$$
\check{r}_{J}=\sum_{\alpha \in \Delta^{+} \backslash \Delta_{J}^{+}} \frac{\langle\alpha, \alpha\rangle}{2} e_{\alpha} \wedge f_{\alpha} .
$$

Observe that $r_{\mathfrak{g}}=r_{J}+\check{r}_{J}$.

4.2. Proposition. Assume that $J \subset \Gamma$ is such that $U_{J}^{+}$is abelian. Then the following properties hold:

(i) The Poisson structure $\pi_{J}$ on $G / P_{J}^{+}$is given by

$$
\pi_{J}=-\chi\left(r_{J}\right) .
$$

In particular, all $L_{J}$-orbits on the flag variety $G / P_{J}^{+}$are complete Poisson subvarieties of $\left(G / P_{J}^{+}, \pi_{J}\right)$.

(ii) Under the identification $\Psi_{J}: \mathfrak{n}_{J}^{-} \stackrel{\cong}{\longrightarrow} B^{-} . P_{J}^{+} \subset G / P_{J}^{+}, \Psi_{J}(x)=\exp (x) P_{J}^{+}$, of $L_{J}$-spaces (where $L_{J}$ acts on the first term by the adjoint action), the restriction of the Poisson structure $\pi_{J}$ to $B^{-} . P_{J}^{+}$corresponds to $-\chi\left(r_{J}\right) \in \Gamma\left(\mathfrak{n}_{J}^{-}, \bigwedge^{2} T \mathfrak{n}_{J}^{-}\right)$. Here $\chi: \bigwedge^{2} \mathfrak{l}_{J} \rightarrow \Gamma\left(\mathfrak{n}_{J}^{-}, \bigwedge^{2} T \mathfrak{n}_{J}^{-}\right)$is derived from the adjoint action of $L_{J}$ on $\mathfrak{n}_{J}^{-}$. 
Proof. (i) For all $u=\exp (x) \in U_{J}^{-}, x \in \mathfrak{n}_{J}^{-}$,

$$
-R_{u}\left(\check{r}_{J}\right)=-L_{u}\left(\operatorname{Ad}_{u}^{-1}\left(\check{r}_{J}\right)\right) \equiv-L_{u}\left(\operatorname{ad}_{x}^{2}\left(\check{r}_{J}\right)\right) / 2 \bmod L_{u}\left(\mathfrak{p}_{J}^{+} \wedge \mathfrak{g}\right)
$$

because $\left[\mathfrak{n}_{J}^{+}, \mathfrak{n}_{J}^{-}\right] \subseteq \mathfrak{l}_{J}$. We have $\operatorname{ad}_{x}^{2}\left(\check{r}_{J}\right)=0$ since for all $y, z \in \mathfrak{n}_{J}^{+}$,

$$
\left\langle\operatorname{ad}_{x}^{2}\left(\check{r}_{J}\right), y \otimes z\right\rangle=\left\langle\operatorname{ad}_{x}^{2}(y), z\right\rangle-\left\langle y, \operatorname{ad}_{x}^{2}(z)\right\rangle=0 .
$$

Therefore (4.3) holds on the open $B^{-}$orbit on $G / P_{J}^{+}$, and thus on the full $G / P_{J}^{+}$ since both sides of (4.3) are algebraic.

The second statement in (i) directly follows from (4.3). It is also a consequence of Theorem 1.1(b) in [19], stating that each $L_{J}$-orbit on $G / P_{J}^{+}$is the intersection of a $P_{J}^{+}$-orbit and a $P_{J}^{-}$-orbit. (The latter are complete Poisson subvarieties of $\left(G / P_{J}^{+}, \pi_{J}\right)$ as was shown in $\S 1.6$. $)$

Part (ii) follows from part (i) by noting that $B^{-} \cdot P_{J}^{+}=U_{J}^{-} \cdot P_{J}^{+}$is $L_{J}$ stable and $\Psi_{J}$ intertwines the actions of $L_{J}$ on $\mathfrak{n}_{J}^{-}$and $B^{-} . P_{J}^{+}$.

4.3. The $L_{J}$-orbits on $G / P_{J}^{+}$were classified by Richardson, Röhrle, and Steinberg [19], and previously the $L_{J}$ orbits on the unipotent radical $U_{J}^{-} \cong B^{-} . P_{J}^{+} \subset G / P_{J}^{+}$ were treated by Wolf [25] and Muller, Rubenthaller, and Schiffmann [15]. We recall the parametrization of the $L_{J}$-orbits on $G / P_{J}^{+}$from [19, Theorem 1.2]. Fix a maximal sequence $\left(\beta_{1}, \ldots, \beta_{k}\right)$ of long roots in $\Delta^{+} \backslash \Delta_{J}^{+}$which are mutually orthogonal. Denote by $u_{-\beta_{i}}$ a nontrivial element in the one-parameter unipotent subgroup of $G$ corresponding to the root $-\beta_{i}$. Denote by $w_{\beta_{i}} \in W$ the reflection corresponding to $\beta_{i}$.

Note that $\left\{w_{\beta_{i}}\right\}_{i=1}^{k}$ mutually commute because $\left\{\beta_{i}\right\}_{i=1}^{k}$ are mutually orthogonal. (The elements $\left\{u_{-\beta_{i}}\right\}_{i=1}^{k}$ also mutually commute because $U_{J}^{-}$is abelian.) Let $\dot{w}_{\beta_{i}}$ be a representative of $w_{\beta_{i}}$ in the normalizer of the maximal torus $H$ of $G$. Finally, set

$$
x_{s t}=\prod_{i=1}^{t} \dot{w}_{\beta_{i}} \prod_{j=t+1}^{s} u_{-\beta_{j}} \quad \text { for } \quad 0 \leq t \leq s \leq k .
$$

Theorem (Richardson-Röhrle-Steinberg [19]). If the parabolic subgroup $P_{J}^{+}$of $G$ has abelian unipotent radical, then $\left\{x_{s t} P_{J}^{+} \mid 0 \leq t \leq s \leq k\right\}$ is a system of representatives for the $L_{J}$-orbits on $G / P_{J}^{+}$.

4.4. Let us recall that $P_{J}^{+}$has an abelian unipotent radical only if it is a maximal parabolic subgroup of $G$. In addition, if $J=\Gamma \backslash\left\{\alpha^{\prime}\right\}$, then $P_{J}^{+}$has abelian unipotent radical if and only the $\alpha^{\prime}$ height of the longest root $\theta$ of $\mathfrak{g}$ is equal to 1 . (We fix this root $\alpha^{\prime}$ for the remainder of this section.) If this condition is satisfied, then the $\alpha^{\prime}$-height of any root $\gamma \in \Delta$ is equal to $0, \pm 1$. Moreover $n_{\alpha^{\prime}}(\gamma)=1,0$, or -1 if $\gamma$ is a root of $\mathfrak{n}_{J}^{+}, \mathfrak{l}_{J}$, or $\mathfrak{n}_{J}^{-}$, respectively (i.e., $\gamma \in \Delta^{+} \backslash \Delta_{J}^{+}, \Delta_{J}$, or $-\left(\Delta^{+} \backslash \Delta_{J}^{+}\right)$).

4.5. We will work with a special maximal set $\left(\beta_{1}, \ldots, \beta_{k}\right)$ of mutually orthogonal long roots in $\Delta^{+} \backslash \Delta_{J}^{+}$. We proceed analogously to the proof of [19, Proposition 2.8], defining inductively $\beta_{i}$ and subsets $\Gamma_{i}$ of the set $\Gamma$ of positive simple roots of $\mathfrak{g}$. Let $\beta_{1}=\theta$ be the highest root of $\mathfrak{g}$ and $\Gamma_{1}=\Gamma$. Assume that for some $i \leq k$, we have already defined $\beta_{i}$ and $\Gamma_{i}$. Let $\widetilde{\Gamma}_{i+1}$ be the set of all roots in $\Gamma_{i}$ that are orthogonal to $\beta_{i}$. (Since $\beta_{i}$ is dominant in the root system defined by $\Gamma_{i}$, all roots in it that are orthogonal to $\beta_{i}$ are combinations of simple roots in $\widetilde{\Gamma}_{i+1}$.) If $k>1$, 
then $\widetilde{\Gamma}_{i+1}$ contains $\alpha^{\prime}$. Denote by $\Gamma_{i+1}$ the connected component of $\widetilde{\Gamma}_{i+1}$ containing $\alpha^{\prime}$. (Here we identify $\Gamma$ with the Dynkin graph of $\mathfrak{g}$ and view $\widetilde{\Gamma}_{i+1}$ as a subgraph of it.) Finally, set $\beta_{i+1}$ to be the highest root of the root system defined by $\Gamma_{i+1}$.

This sequence has the properties that

$$
\operatorname{supp} \beta_{j} \subseteq \operatorname{supp} \beta_{i}=\Gamma_{i} \text { for } j \geq i
$$

(cf. $\S 0.7$ ) and that $\beta_{i}$ is the longest root in the root system defined by $\Gamma_{i}$.

For simplicity of the exposition, we set

$$
u_{-\beta_{i}}=\exp \left(f_{\beta_{i}}\right)
$$

but we note that all proofs work for general $u_{-\beta_{i}}$.

We will also use special representatives for the reflections $w_{\beta_{i}} \in W$ in the normalizer of the maximal torus $H$ of $G$ (and will thus omit the dot on top of them). Set

$$
w_{\beta_{i}}=\exp \left(e_{\beta_{i}}\right) \exp \left(-f_{\beta_{i}}\right) \exp \left(e_{\beta_{i}}\right) .
$$

This normalization is not necessary for the proof of the main result in Theorem 4.6, but it simplifies the exposition. Since $\beta_{i}$ and $\beta_{j}$ are orthogonal for $i \neq j$ and $\beta_{i}+\beta_{j}$ is not a root of $\mathfrak{g}$, we have that $w_{\beta_{i}}\left(\beta_{j}\right)=0$ and $\left[f_{\beta_{i}}, f_{\beta_{j}}\right]=0$, and consequently

$$
\begin{aligned}
{\left[e_{\beta_{i}}, f_{\beta_{j}}\right] } & =0, & & \operatorname{Ad}_{w_{\beta_{i}}}\left(f_{\beta_{j}}\right)=f_{\beta_{j}}, \\
\operatorname{Ad}_{w_{\beta_{i}}}\left(u_{-\beta_{j}}\right) & =u_{-\beta_{j}}, & & \operatorname{Ad}_{w_{\beta_{i}}}\left(e_{\beta_{j}}\right)=e_{\beta_{j}}
\end{aligned}
$$

for the special representatives (4.6) of $w_{\beta_{i}}$.

The first main result in this section is contained in the following Theorem.

4.6. Theorem. If $P_{J}^{+}$is a parabolic subgroup with abelian unipotent radical in the complex simple algebraic group $G$, then the Poisson structure $\pi_{J}$ vanishes at the base points $x_{s t} P_{J}^{+}$of the $L_{J}$-orbits on $G / P_{J}^{+}(0 \leq t \leq s \leq k)$. Therefore, all $L_{J^{-}}$ orbits on $G / P_{J}^{+}$are complete Poisson subvarieties of $\left(G / P_{J}^{+}, \pi_{J}\right)$ and are quotients of $\left(L_{J}, \pi_{L_{J}}\right) ; c f$. [3, Theorem 1.8].

Even in the case of Grassmannians, Theorem 4.6 contains new information, compared to Part I [3], where we dealt with the open $B^{-}$-orbits on Grassmannians.

Let us note that Theorem 4.6 is not valid for an arbitrary maximal set $\left(\beta_{1}, \ldots, \beta_{k}\right)$ of mutually orthogonal long roots of $\Delta^{+} \backslash \Delta_{J}^{+}$. For instance, in the case of $A_{4}$ and $\alpha^{\prime}=\epsilon_{3}-\epsilon_{2}$, take $\beta_{1}=\epsilon_{3}-\epsilon_{1}$ and $\beta_{2}=\epsilon_{4}-\epsilon_{2}$. Then $\pi_{J}$ does not vanish at $u_{-\beta_{1}} u_{-\beta_{2}} P_{J}^{+}$. The easiest way to see this is to note that the Poisson structure $\pi_{2,2}$ on the matrix affine Poisson space $M_{2,2}$ (cf. [3, eq. (1.7)]) does not vanish at $\left[\begin{array}{ll}0 & 1 \\ 1 & 0\end{array}\right]$ and then to use the embedding from [3, Proposition 3.4].

For the proof of Theorem 4.6, we will need several lemmas.

4.7. Lemma. Assume that $\beta$ is a long root in $\Delta^{+} \backslash \Delta_{J}^{+}$and set

$$
w_{\beta}=\exp \left(e_{\beta}\right) \exp \left(-f_{\beta}\right) \exp \left(e_{\beta}\right) \text {. }
$$

Let $\alpha$ be any root of $\mathfrak{g}$ and $y_{\alpha} \in \mathfrak{g}$ a nonzero vector in the corresponding root space. If $\left[f_{\beta}, y_{\alpha}\right] \neq 0$, then $w_{\beta}(\alpha)=\alpha-\beta$ and

$$
\left[f_{\beta}, y_{\alpha}\right]=-\operatorname{Ad}_{w_{\beta}}\left(y_{\alpha}\right) .
$$

Proof. First, there is no root $\gamma$ of $\mathfrak{g}$ such that $\gamma-\beta$ and $\gamma-2 \beta$ are roots as well. For if this happens, then the $\alpha^{\prime}$ height of $\gamma$ needs to be equal to 1, i.e., $\gamma \in \Delta^{+} \backslash \Delta_{J}^{+}$, because the $\alpha^{\prime}$ heights of all roots of $\mathfrak{g}$ are 0 or \pm 1 . Consequently, 
$\gamma+\beta$ would not be a root and $\left\langle\gamma, \beta^{\vee}\right\rangle \geq 2$. This would be a contradiction, since $\left|\left\langle\gamma, \beta^{\vee}\right\rangle\right| \leq 1$ because $\beta$ is a long root. From this we get that the only roots of $\mathfrak{g}$ of the form $\alpha+i \beta$ for $i \in \mathbb{Z}$ are $\alpha$ and $\alpha-\beta$; thus $w_{\beta}(\alpha)=\alpha-\beta \operatorname{ad}_{e_{\beta}}\left(x_{\alpha}\right)=0$, and $\operatorname{ad}_{f_{\beta}}^{2}\left(x_{\alpha}\right)=0$. Consequently, $\operatorname{Span}\left\{y_{\alpha},\left[f_{\beta}, y_{\alpha}\right]\right\}$, under the adjoint action, is isomorphic to the vector representation of the $\mathfrak{s l}_{2}$ triple $\left\{e_{\beta}, \beta^{\vee}=\left[e_{\beta}, f_{\beta}\right], f_{\beta}\right\}$. By a standard computation, in this basis $w_{\beta}$ acts by $\left[\begin{array}{cc}0 & 1 \\ -1 & 0\end{array}\right]$, so $\operatorname{Ad}_{w_{\beta}}\left(y_{\alpha}\right)=-\left[f_{\beta}, y_{\alpha}\right]$.

4.8. Lemma. If $\alpha \in \Delta_{J}^{+}$is such that

$$
\left[f_{\beta_{i}}, e_{\alpha}\right] \neq 0 \text { and }\left[f_{\beta_{j}}, f_{\alpha}\right] \neq 0
$$

for some $i \neq j \leq k$, then $\beta_{i}-\alpha-\beta_{j} \in \Delta_{J}^{+}$and

$$
\left[f_{\beta_{i}}, e_{\alpha}\right] \wedge\left[f_{\beta_{j}}, f_{\alpha}\right]+\left[f_{\beta_{i}}, e_{\beta_{i}-\alpha-\beta_{j}}\right] \wedge\left[f_{\beta_{j}}, f_{\beta_{i}-\alpha-\beta_{j}}\right]=0 .
$$

Proof. It follows from Lemma 4.7 that

$$
w_{\beta_{i}}(\alpha)=\alpha-\beta_{i} \quad \text { and } \quad w_{\beta_{j}}(-\alpha)=-\alpha-\beta_{j} .
$$

Since $\beta_{i}$ and $\beta_{j}$ are orthogonal, $w_{\beta_{j}}\left(\beta_{i}\right)=0$ and

$$
w_{\beta_{j}} w_{\beta_{i}}(\alpha)=w_{\beta_{j}}\left(\alpha-\beta_{i}\right)=\alpha+\beta_{j}-\beta_{i} .
$$

Therefore $\beta_{i}-\alpha-\beta_{j}$ is a root of $\mathfrak{g}$. Because $\alpha-\beta_{i}$ is a root of $\alpha^{\prime}$ height equal to -1 , it belongs to $-\left(\Delta^{+} \backslash \Delta_{J}^{+}\right)$. As a consequence of this, $\operatorname{supp} \alpha \subset \operatorname{supp} \beta_{i}=\Gamma_{i}$; recall $\S 4.5$. If $i<j$, then $\operatorname{supp} \alpha \subset \Gamma_{i} \subset \Gamma_{j}$, and the fact that $\alpha+\beta_{j}$ is a root would contradict the property that $\beta_{j}$ is the highest root of the root system of $\Gamma_{j}$; cf. $\S 4.5$. Thus, $i>j$ and $\alpha+\beta_{j}$ is a positive root in the root system of $\Gamma_{i}$. Since $\beta_{i}$ is the highest root in this root system, $\beta_{i}-\alpha-\beta_{j}$ needs to be a positive root.

Because $\operatorname{Ad}_{w_{\beta_{j}} w_{\beta_{i}}}$ preserves the bilinear form $\langle.,$.$\rangle on \mathfrak{g}$,

$$
\operatorname{Ad}_{w_{\beta_{j}} w_{\beta_{i}}}\left(e_{\alpha}\right)=a f_{\beta_{i}-\alpha-\beta_{j}} \text { and } \operatorname{Ad}_{w_{\beta_{j}} w_{\beta_{i}}}\left(f_{\alpha}\right)=a^{-1} e_{\beta_{i}-\alpha-\beta_{j}}
$$

for some $a \in \mathbb{C}^{\times}$. Equation (4.6) implies that $\operatorname{Ad}_{w_{\beta_{i}}}\left(f_{\beta_{i}}\right)=-e_{\beta_{i}}$. Combining the above facts and using the fact that $\operatorname{Ad}_{w_{\beta_{j}}}\left(e_{\beta_{i}}\right)=e_{\beta_{i}}$ (cf. §4.5) leads to

$$
\operatorname{Ad}_{w_{\beta_{j}} w_{\beta_{i}}}\left(\left[f_{\beta_{i}}, e_{\alpha}\right]\right)=-a\left[e_{\beta_{i}}, f_{\beta_{i}-\alpha-\beta_{j}}\right] .
$$

Thus, $\left[e_{\beta_{i}}, f_{\beta_{i}-\alpha-\beta_{j}}\right] \neq 0$, and consequently $\left[f_{\beta_{i}}, e_{\beta_{i}-\alpha-\beta_{j}}\right] \neq 0$. Applying Lemma 4.7 and (4.8) twice, we get that

$$
\begin{aligned}
{\left[f_{\beta_{i}}, e_{\beta_{i}-\alpha-\beta_{j}}\right] } & =-\operatorname{Ad}_{w_{\beta_{i}}}\left(e_{\beta_{i}-\alpha-\beta_{j}}\right)=-a \operatorname{Ad}_{w_{\beta_{i}}^{2} w_{\beta_{j}}}\left(f_{\alpha}\right) \\
& =a \operatorname{Ad}_{w_{\beta_{j}}}\left(f_{\alpha}\right)=-a\left[f_{\beta_{j}}, f_{\alpha}\right] .
\end{aligned}
$$

In the third equality, we used the fact that $\operatorname{Ad}_{w_{\beta_{j}}}\left(f_{\alpha}\right)$ is the lowest weight vector for the vector representation of the $\mathfrak{s l}_{2}$ triple $\left\{e_{\beta_{i}}, \beta_{i}^{\vee}=\left[e_{\beta_{i}}, f_{\beta_{i}}\right], f_{\beta_{i}}\right\}$ (under the adjoint action), and in this representation $w_{\beta_{i}}^{2}$ acts by - id; recall (4.6). Analogously,

$$
\begin{aligned}
{\left[f_{\beta_{j}}, f_{\beta_{i}-\alpha-\beta_{j}}\right] } & =-\operatorname{Ad}_{w_{\beta_{j}}}\left(f_{\beta_{i}-\alpha-\beta_{j}}\right)=-a^{-1} \operatorname{Ad}_{w_{\beta_{i}} w_{\beta_{j}}^{2}}\left(e_{\alpha}\right) \\
& =a^{-1} \operatorname{Ad}_{w_{\beta_{i}}}\left(e_{\alpha}\right)=-a^{-1}\left[f_{\beta_{i}}, e_{\alpha}\right] .
\end{aligned}
$$

Hence,

$$
\begin{aligned}
{\left[f_{\beta_{i}}, e_{\beta_{i}-\alpha-\beta_{j}}\right] \wedge\left[f_{\beta_{j}}, f_{\beta_{i}-\alpha-\beta_{j}}\right] } & =\left(-a\left[f_{\beta_{j}}, f_{\alpha}\right]\right) \wedge\left(-a^{-1}\left[f_{\beta_{i}}, e_{\alpha}\right]\right) \\
& =-\left[f_{\beta_{i}}, e_{\alpha}\right] \wedge\left[f_{\beta_{j}}, f_{\alpha}\right] .
\end{aligned}
$$


4.9. Lemma. For all $0 \leq t \leq s \leq k$,

$$
\operatorname{Ad}_{u_{-\beta_{t+1}} \ldots u_{-\beta_{s}}}^{-1}\left(r_{J}\right)=r_{J}-\sum_{j=t+1}^{s} \sum_{\alpha \in \Delta_{J}^{+}} \frac{\langle\alpha, \alpha\rangle}{2}\left(\left[f_{\beta_{j}}, e_{\alpha}\right] \wedge f_{\alpha}+e_{\alpha} \wedge\left[f_{\beta_{j}}, f_{\alpha}\right]\right) .
$$

Proof. For arbitrary $0 \leq i \leq k$ and $0 \leq j \leq k$ and $\alpha \in \Delta_{J}$, the sum $\alpha+\beta_{i}+\beta_{j}$ is not a root of $\mathfrak{g}$ since its $\alpha^{\prime}$ height is -2 . Taking into account that $u_{-\beta_{j}}=\exp \left(f_{\beta_{j}}\right)$, we get

$$
\begin{aligned}
& \operatorname{Ad}_{u_{-\beta_{t+1}} \ldots u_{-\beta_{s}}}^{-1}\left(r_{J}\right) \\
& \quad=\sum_{\alpha \in \Delta_{J}^{+}}\left(e_{\alpha}-\left[f_{\beta_{t+1}}, e_{\alpha}\right]-\ldots-\left[f_{\beta_{s}}, e_{\alpha}\right]\right) \wedge\left(f_{\alpha}-\left[f_{\beta_{t+1}}, f_{\alpha}\right]-\ldots-\left[f_{\beta_{s}}, f_{\alpha}\right]\right) .
\end{aligned}
$$

The Lemma will follow if we show that for all $s \leq i, j \leq k$,

$$
\sum_{\alpha \in \Delta_{J}^{+}}\left[f_{\beta_{i}}, e_{\alpha}\right] \wedge\left[f_{\beta_{j}}, f_{\alpha}\right]=0
$$

This is a corollary of Lemma 4.8 , which implies that all $\alpha \in \Delta_{J}^{+}$for which $\left[f_{\beta_{i}}, e_{\alpha}\right] \neq$ 0 and $\left[f_{\beta_{j}}, f_{\alpha}\right] \neq 0$ can be grouped in pairs such the sum of the corresponding expressions $\left[f_{\beta_{i}}, e_{\alpha}\right] \wedge\left[f_{\beta_{j}}, f_{\alpha}\right]$ will be equal to 0 .

In the setting of $\S 4.3$, for each $0 \leq t \leq k$ set

$$
w_{t}=\prod_{i=1}^{t} w_{i} .
$$

The same notation will be used for the representative of $w_{t}$ in the normalizer of $H$ in $G$ which is the product of the representatives (4.6).

4.10. Proof of Theorem 4.6. We will prove that

$$
\left.\operatorname{Ad}_{w_{t}}^{-1}\left(\left[f_{\beta_{j}}, e_{\alpha}\right]\right) \wedge f_{\alpha}\right) \in \mathfrak{p}_{J}^{+} \wedge \mathfrak{g}, \quad \text { for } \alpha \in \Delta_{J}^{+}, 0 \leq t<j \leq k .
$$

Analogously, one shows that

$$
\operatorname{Ad}_{w_{t}}^{-1}\left(e_{\alpha} \wedge\left[f_{\beta_{j}}, f_{\alpha}\right]\right) \in \mathfrak{p}_{J}^{+} \wedge \mathfrak{g}, \quad \text { for } \alpha \in \Delta_{J}^{+}, 0 \leq t<j \leq k .
$$

It is clear that

$$
\operatorname{Ad}_{w_{t}}^{-1}\left(e_{\alpha} \wedge f_{\alpha}\right) \in \mathfrak{n}^{-} \wedge \mathfrak{n}^{+}, \quad \text { for } \alpha \in \Delta_{J}^{+}, 0 \leq t \leq k .
$$

Lemma $4.9,(4.10)-(4.12)$, and the commutativity of $w_{t}$ and $u_{-\beta_{t+1}} \ldots u_{-\beta_{s}}$ (cf. $\S 4.5)$ imply that

$$
\operatorname{Ad}_{x_{s t}}\left(r_{J}\right) \in \mathfrak{p}_{J}^{+} \wedge \mathfrak{g}, \quad \text { for } 0 \leq t \leq s \leq k,
$$

which is equivalent to the vanishing of $\pi_{J}$ at $x_{s t} P_{J}^{+}$.

Thus, we are left with showing (4.10). We will make use of the following fact [19, Lemma $2.10(\mathrm{~b})]$ :

(*) For $\gamma \in-\left(\Delta^{+} \backslash \Delta_{J}^{+}\right)$, the set of all $\beta_{i}, 0 \leq i \leq t$, not orthogonal to $\gamma$ has cardinality 0,1 , or 2 , and accordingly $w_{t}(\gamma) \in-\left(\Delta^{+} \backslash \Delta_{J}^{+}\right), \Delta_{J}$, or $\Delta^{+} \backslash \Delta_{J}^{+}$.

If $w_{t}^{-1}(-\alpha) \notin-\left(\Delta^{+} \backslash \Delta_{J}^{+}\right)$, then $\operatorname{Ad}_{w_{t}}^{-1}\left(f_{\alpha}\right) \in \mathfrak{p}_{J}^{+}$, and we are done. If $w_{t}(\alpha)^{-1} \in$ $-\left(\Delta^{+} \backslash \Delta_{J}^{+}\right)$, then applying $\left(^{*}\right)$ for $\gamma=w_{t}^{-1}(-\alpha)$ one gets that there exists $0 \leq i \leq t$ such that $w_{t}^{-1}(-\alpha)=w_{\beta_{i}}(-\alpha)=-\alpha-\beta_{i}$. This means that $\left\langle\beta_{i}^{\vee}, \alpha\right\rangle=-1$, and 
consequently $\left\langle\beta_{i}^{\vee}, \alpha-\beta_{j}\right\rangle=-1\left(\left\langle\beta_{i}, \beta_{j}\right\rangle=0\right.$ since $\left.j>t \geq i\right)$. If $\left[f_{\beta_{j}}, e_{\alpha}\right]=0$, then we are done. If $\left[f_{\beta_{j}}, e_{\alpha}\right] \neq 0$, then using $(*)$ again, this time for $\gamma=\alpha-\beta_{j}$, we get $w_{t}^{-1}\left(\alpha-\beta_{j}\right) \notin-\left(\Delta^{+} \backslash \Delta_{J}^{+}\right)$; therefore $\left.\operatorname{Ad}_{w_{t}}^{-1}\left[f_{\beta_{j}}, e_{\alpha}\right]\right) \in \mathfrak{p}_{J}^{+}$. This establishes (4.10) and thus the Theorem.

4.11. In the last part of this section $(\S \S 4.11-4.13)$, for a parabolic subgroup $P_{J}^{+}$ with abelian unipotent radical, we characterize the symplectic leaves of $\left(G, \pi_{J}^{+}\right)$ within each $L_{J}$-orbit. First we recall some facts on minimal length representatives in Weyl groups. For two subsets $I$ and $J$ of the set of positive simple roots $\Gamma$ of $G$ denote by ${ }^{I} W_{\min }$ and ${ }^{I} W_{\min }^{J}$ the set of (unique) minimal length representatives of the cosets in $W_{I} \backslash W$ and $W_{I} \backslash W / W_{J}$. Recall the following standard facts; see e.g. 4. Lemma 4.3].

Lemma. For arbitrary $I, J \subset \Gamma$ the following hold:

(i) Every element of $W_{\min }^{J}$ can be uniquely represented as a product $v w$ for some $w \in{ }^{I} W_{\min }^{J}$ and $v \in W_{I} \cap W_{\min }^{I \cap w(J)}$.

(ii) Every element of $W$ can be uniquely represented as a product $v_{1} w v_{2}$ for some $w \in{ }^{I} W_{\min }^{J}, v_{2} \in W_{J}$ and $v_{1} \in W_{I} \cap W_{\min }^{I \cap w(J)}$.

4.12. We will need the following results from [19]; recall (4.9).

Theorem (Richardson-Röhrle-Steinberg). If $P_{J}^{+} \subset G$ has abelian unipotent radical, then the following hold:

(i) For any given $P_{J}^{+}$-orbit and any given $P_{J}^{-}$-orbit on $G / P_{J}^{+}$, the two orbits are either disjoint or else intersect in a single $L_{J}$-orbit.

(ii) The set $\left\{w_{s}\right\}_{s=0}^{k}$ coincides with ${ }^{J} W_{\min }^{J}$ and thus $G / P_{J}^{+}=\bigsqcup_{0 \leq s \leq k} P_{J}^{+} . w_{s} P_{J}^{+}$ $=\bigsqcup_{0 \leq s \leq k} P_{J}^{-} \cdot w_{s} P_{J}^{+}$.

(iii) For all $0 \leq t \leq s \leq k$, we have

$$
L_{J} \cdot x_{s t} P_{J}^{+}=P_{J}^{-} \cdot w_{t} P_{J}^{+} \cap P_{J}^{+} \cdot w_{s} P_{J}^{+}
$$

cf. (4.9).

Part (i) is [19, Theorem 1.1 (b)], part (ii) is [19, Proposition 2.11], and part (iii) is [19, Lemma $3.5(\mathrm{~d})]$. We will illustrate the inclusion

$$
L_{J} \cdot x_{s t} P_{J}^{+} \subset P_{J}^{-} \cdot w_{t} P_{J}^{+} \cap P_{J}^{+} \cdot w_{s} P_{J}^{+} .
$$

Since $\dot{w}_{\beta_{i}}$ and $u_{-\beta_{j}}$ commute for $i \neq j$ (cf. $\S 4.5$ ),

$$
L_{J} . x_{s t} P_{J}^{+}=L_{J} \prod_{j=t+1}^{s} u_{-\beta_{j}} \cdot \prod_{i=1}^{t} \dot{w}_{i} P_{J}^{+} \subset P_{J}^{-} w_{t} P_{J}^{+} .
$$

Because $\left[e_{\beta_{i}}, f_{\beta_{j}}\right]=0$ for $i \neq j$ and $w_{\beta_{i}}^{2}=1$,

$$
\begin{aligned}
L_{J} . x_{s t} P_{J}^{+} & =L_{J} \prod_{i=1}^{t} \exp \left(e_{\beta_{i}}\right)\left(\prod_{i=1}^{t} \exp \left(-e_{\beta_{i}}\right) \exp \left(f_{\beta_{i}}\right) \exp \left(-e_{\beta_{i}}\right)\right) \prod_{j=t+1}^{s} w_{\beta_{j}} P_{J}^{+} \\
& \subset P_{J}^{+} . w_{s} P_{J}^{+} .
\end{aligned}
$$

The second main result of this section is contained in the following Theorem. 
4.13. Theorem. Assume that $P_{J}^{+}$is a parabolic subgroup of $G$ with abelian unipotent radical. For all $0 \leq s \leq t \leq k$, the $H$-orbits of symplectic leaves of $\left(L_{J} . x_{s t} P_{J}^{+},\left.\pi_{J}\right|_{L_{J} . x_{s t} P_{J}^{+}}\right)$are $\mathcal{S}_{\left(v_{1} w_{t} w_{0}^{J}, v_{2} w_{s} v_{3}\right)}(c f .(1.9))$ for unique

$$
v_{3} \in W_{J}, \quad v_{1} \in W_{J} \cap W_{\min }^{J \cap w_{t}(J)}, \quad v_{2} \in W_{J} \cap W_{\min }^{J \cap w_{s}(J)}
$$

such that $v_{1} w_{t} w_{\circ}^{J} \leq v_{2} w_{s} v_{3}$.

As an example, let us note that the $L_{J}$-orbits inside the open $B^{-}$-orbit on $G / P_{J}^{+}$are the orbits $L_{J} \cdot x_{0 s} P_{J}^{+}$, for $0 \leq s \leq k$. Theorem 4.13 implies that the symplectic leaves of $\left(L_{J} . x_{0 s} P_{J}^{+},\left.\pi_{J}\right|_{L_{J} . x_{0 s} P_{J}^{+}}\right)$are $\mathcal{S}_{\left(w_{o}^{J}, v_{2} w_{t} v_{3}\right)}^{J}$ for some $v_{3} \in W_{J}$ and $v_{2} \in W_{J} \cap W_{\min }^{J \cap w_{s}(J)}$ such that $w_{\circ}^{J} \leq v_{2} w_{s} v_{3}$.

Proof. Because $\mathcal{U}_{\dot{w}_{1}, w_{2}} \subset B^{-} w_{1} \cap B^{+} w_{2} B^{+}$(recall (0.6)), we have

$$
\mathcal{S}_{w_{1}, w_{2}}^{J}=\mathcal{U}_{\dot{w}_{1}, w_{2}} \cdot P_{J}^{+} \subset B^{-} x_{w_{1}}^{J} \cap B^{+} x_{w_{2}}^{J} \subset P_{J}^{-} x_{w_{1}}^{J} \cap P_{J}^{+} x_{w_{2}}^{J} .
$$

It follows from Lemma 4.11, Theorem 4.12 (ii), and (1.3) that every element of $\Omega^{J}$ can be uniquely represented in the form $\left(v_{1} w_{t} w_{\circ}^{J}, v_{2} w_{s} v_{3}\right)$ for some $0 \leq s, t \leq k$ and $v_{3}, v_{1}, v_{2}$ as in (4.13) such that $v_{1} w_{t} w_{\circ}^{J} \leq v_{2} w_{s} v_{3}$. For such a pair, we have

$$
\mathcal{S}_{\left(v_{1} w_{t} w_{\circ}^{J}, v_{2} w_{s} v_{3}\right)}^{J} \subset P_{J}^{-} \cdot v_{1} w_{t} w_{\circ}^{J} P_{J}^{+} \cap P_{J}^{+} \cdot v_{2} w_{s} v_{3} P_{J}^{+}=P_{J}^{+} \cdot w_{t} P_{J}^{+} \cap P_{J}^{-} \cdot w_{s} P_{J}^{+} .
$$

Theorem 4.12 implies that the last intersection is nontrivial only if $t \leq s$, in which case it is equal to $L_{J} \cdot x_{t s} P_{J}^{+}$. This completes the proof of the Theorem.

\section{The open $B^{-}$-orbits in compact Hermitian symmetric spaces}

In this section, we treat in detail the restriction of the Poisson structure $\pi_{J}$ to the open $B^{-}$-orbit of $G / P_{J}^{+}$in the case when $G$ is a complex simple group and $P_{J}^{+}$is a parabolic subgroup with abelian unipotent radical. First, in $\S 5.1$, we obtain general formulas for $\left.\pi_{J}\right|_{B^{-} . P_{J}^{+}}$. Then, in $\S \S 5.3-5.6$, we use it to derive explicit formulas for all classical groups and show that all such Poisson structures are quasiclassical limits of interesting classes of quadratic algebras, of the type known as quantized coordinate rings of classical varieties. (See, e.g., [10] for a general survey of quantized coordinate rings.) In $§ 5.7$, we show that the $E_{6}$ case gives rise to a new quadratic Poisson structure on a 16 dimensional affine space, related to a half-spin representation of $\mathfrak{s o}_{10}$.

5.1. For a general reductive Lie algebra $\mathfrak{g}$ with fixed dual Borel subalgebras $\mathfrak{b}^{ \pm}$ as in the Introduction, consider an irreducible representation $V_{\mathfrak{g}}^{\lambda}$ of $\mathfrak{g}$ with highest weight $\lambda$. Define the bivector field

$$
\pi_{\mathfrak{g}}^{\lambda}=\chi\left(r_{\mathfrak{g}}\right) \in \Gamma\left(\left(V_{\mathfrak{g}}^{\lambda}\right)^{*}, \bigwedge^{2} T\left(V_{\mathfrak{g}}^{\lambda}\right)^{*}\right)
$$

(cf. (0.1) and $\S 0.2$ ), where $\chi$ is derived from the action of $\mathfrak{g}$ on $\left(V_{\mathfrak{g}}^{\lambda}\right)^{*}$. If $\pi_{\mathfrak{g}}^{\lambda}$ is Poisson, then the corresponding Poisson bracket on the algebra of regular functions on $\left(V_{\mathfrak{g}}^{\lambda}\right)^{*}$, identified with the symmetric algebra $S\left(V_{\mathfrak{g}}^{\lambda}\right)$, is induced by

$$
\left\{v_{1}, v_{2}\right\}=m\left(r_{\mathfrak{g}}\left(v_{1} \otimes v_{2}\right)\right), \quad v_{1}, v_{2} \in V_{\mathfrak{g}}^{\lambda},
$$

where $m: V_{\mathfrak{g}}^{\lambda} \otimes V_{\mathfrak{g}}^{\lambda} \rightarrow S\left(V_{\mathfrak{g}}^{\lambda}\right)$ is the multiplication map, $m\left(v_{1}, v_{2}\right)=v_{1} v_{2}$. 
In the setting of $\S 0.2$, for $\alpha \in \Delta_{J}^{+}$and $\beta \in \Delta^{+} \backslash \Delta_{J}^{+}$set

$$
\left[e_{\alpha}, e_{\beta}\right]=N_{\alpha, \beta} e_{\alpha+\beta}, \quad\left[f_{\alpha}, e_{\beta}\right]=N_{-\alpha, \beta} e_{-\alpha+\beta}, \quad N_{\alpha, \beta}, N_{-\alpha, \beta} \in \mathbb{C} .
$$

For a Lie algebra $\mathfrak{g}$, we will denote by $\mathfrak{g}^{\prime}=[\mathfrak{g}, \mathfrak{g}]$ its derived subalgebra.

Finally, for arbitrary $J \subset \Gamma$, denote by $\left.\theta\right|_{J}$ the restriction of the highest root of $\mathfrak{g}$ to (a dominant weight of) $\mathfrak{l}_{J}$.

Proposition. Assume that $G$ is a complex simple algebraic group and $P_{J}^{+}$is a parabolic subgroup of $G$ with abelian unipotent radical. Then the following hold:

(i) Under the adjoint action of $\mathfrak{l}_{J}$, the space $\mathfrak{n}_{J}^{+}$is an irreducible representation of $\mathfrak{l}_{J}^{\prime}$ with highest weight $\left.\theta\right|_{J}$. The restriction of the Poisson structure $\pi_{J}$ to $B^{-} . P_{J}^{+} \subset$ $G / P_{J}^{+}$, identified with $\mathfrak{n}_{J}^{-}$by $x \mapsto \exp (x) P_{J}^{+}$, coincides with $-\pi_{\mathfrak{l}_{J}^{\prime}}^{\left.\theta\right|_{J}} ; c f$. (5.1)-(5.2).

(ii) The Poisson structure on $\mathfrak{n}_{J}^{-}=\left\{\sum_{\beta \in \Delta^{+} \backslash \Delta_{J}^{+}} y_{\beta} f_{\beta} \mid y_{\beta} \in \mathbb{C}\right\} \cong \mathbb{A}^{\left|\Delta^{+}\right|-\left|\Delta_{J}^{+}\right|}$ from the first part is also is given by

$$
\begin{array}{r}
\left\{y_{\beta}, y_{\gamma}\right\}=\sum_{\alpha \in \Delta_{J}^{+}} \frac{\langle\alpha, \alpha\rangle}{2}\left(-N_{\alpha, \beta} N_{-\alpha, \gamma} y_{\alpha+\beta} y_{-\alpha+\gamma}+N_{-\alpha, \beta} N_{\alpha, \gamma} y_{-\alpha+\beta} y_{\alpha+\gamma}\right), \\
\beta, \gamma \in \Delta^{+} \backslash \Delta_{J}^{+} .
\end{array}
$$

Proof. The first statement of part (i) is well known. The simplest way to show it here is to observe that $L_{J}$ has finitely many orbits on $\mathfrak{n}_{J}^{-}$(acting by the adjoint action) because it has finitely many orbits on $G / P_{J}^{+}$. This implies that $\mathfrak{n}_{J}^{-}$is an irreducible representation of $\mathfrak{l}_{J}^{\prime}$ (under the adjoint action) and it must have highest weight $\left.\theta\right|_{J}$.

The second statement in part (i) now follows from the first one, Proposition 4.2(ii), and the definition (5.1).

Part (ii) is an immediate corollary of part (i); see (5.2).

5.2. Assume that the positive simple roots $\alpha_{1}, \ldots, \alpha_{N}$ of the simple algebraic group $G$ are enumerated as in [1. Recall that only maximal parabolic subgroups $P_{J}^{+}$of $G$ can have an abelian unipotent radical. Moreover, those are exactly the parabolic subgroups $P_{\Gamma \backslash\left\{\alpha_{m}\right\}}^{+}$for which the simple root $\alpha_{m}$ appears with multiplicity 1 in the expansion of the highest root $\theta$ of $\mathfrak{g}$ in terms of the positive simple roots, i.e., the $\alpha_{m}$ height of $\theta$ is equal to 1 . In other words, $\alpha_{m}$ should be a cominuscule root. This leads to the following choices for $\alpha_{m}$ according to the type of $G$ :

$$
\begin{array}{ll}
A_{N} & \alpha_{1}, \ldots, \alpha_{N}, \\
B_{N} & \alpha_{1}, \\
C_{N} & \alpha_{N}, \\
D_{N} & \alpha_{1}, \alpha_{N-1}, \alpha_{N}, \\
E_{6} & \alpha_{1}, \alpha_{6}, \\
E_{7} & \alpha_{7} .
\end{array}
$$

For the other types $\left(E_{8}, F_{4}, G_{2}\right)$, there are no parabolic subgroups with abelian unipotent radicals. Below we will denote by $\omega_{m}$ the $m$-th fundamental weight of $G$. 
5.3. $A_{N-1}$ case. In this case, $\mathfrak{g}=\mathfrak{s l}_{N}$,

$$
\theta=\alpha_{1}+\ldots+\alpha_{N}=\omega_{1}+\omega_{N}
$$

and all simple roots $\alpha_{m}(m=1, \ldots, N)$ are cominuscule. The derived subalgebra of the Levi factor of the parabolic subalgebra $\mathfrak{p}_{\Gamma \backslash\left\{\alpha_{m}\right\}}^{+}$is $\mathfrak{l}_{\Gamma \backslash\left\{\alpha_{m}\right\}}^{\prime} \cong \mathfrak{s l}_{m} \oplus \mathfrak{s l}_{N-m+1}$. Under the adjoint action of $\mathfrak{l}_{\Gamma \backslash\left\{\alpha_{m}\right\}}^{\prime}$, the nilradical $\mathfrak{n}_{\Gamma \backslash\left\{\alpha_{m}\right\}}^{-}$is identified with $V_{\mathfrak{s l}_{m}}^{\omega_{1}} \otimes$ $\left(V_{\mathfrak{s l}_{N-m+1}}^{\omega_{1}}\right)^{*}$, where $V_{\mathfrak{s l}_{l}}^{\omega_{1}}$ denotes the vector representation of $\mathfrak{s l}_{l}$. In Part I, we showed that, after applying the twist [3, eq. (3.14)], the induced Poisson structure on $\mathfrak{n}_{\Gamma \backslash\left\{\alpha_{m}\right\}}^{-}$corresponds to the standard quadratic Poisson structure on the space of rectangular matrices $M_{N-m+1, m}$ (cf. [3, eq. (1.7)]). It is the quasiclassical limit of the quadratic algebra of quantum matrices.

5.4. $B_{N+1}$ case. In this case, $\mathfrak{g} \cong \mathfrak{s o}_{2 N+1}$,

$$
\theta=\alpha_{1}+2 \alpha_{2}+\ldots+2 \alpha_{N}=\omega_{2},
$$

and the only root that is cominuscule is $\alpha_{1}$. Furthermore, $\mathfrak{l}_{\Gamma \backslash\left\{\alpha_{1}\right\}}^{\prime} \cong \mathfrak{s o}_{2 N-1}$, and under the adjoint action the nilradical $\mathfrak{n}_{\Gamma \backslash\left\{\alpha_{1}\right\}}^{-}$corresponds to the vector representation $V_{\mathfrak{s o}_{2 N-1}}^{\omega_{1}}$ of $\mathfrak{s o}_{2 N-1}$. If we identify

$$
\begin{aligned}
\mathfrak{n}_{\Gamma \backslash\left\{\alpha_{1}\right\}}^{-} \cong\left\{\sum_{i=2}^{N} x_{i}\left(E_{N+i, 1}-E_{N+1, i}\right)+\sum_{j=2}^{N} y_{j}\left(E_{j, 1}-E_{N+1, N+j}\right)\right. & \\
& \left.+z\left(E_{2 N+1,1}-E_{N+1,2 N+1}\right) \mid x_{i}, y_{j}, z \in \mathbb{C}\right\}=\mathbb{A}^{2 N-1},
\end{aligned}
$$

then the induced Poisson structure on $\mathfrak{n}_{\Gamma \backslash\left\{\alpha_{1}\right\}}^{-}$is given by

$$
\begin{aligned}
2\left\{x_{i}, x_{j}\right\} & =x_{i} x_{j}, & & i<j, \\
2\left\{y_{i}, y_{j}\right\} & =-y_{i} y_{j}, & & i<j, \\
2\left\{z, x_{i}\right\} & =-z x_{i}, & & \text { for all } z, \\
2\left\{z, y_{j}\right\} & =z y_{j}, & & \text { for all } j, \\
2\left\{x_{i}, y_{j}\right\} & =x_{i} y_{j}, & & i \neq j, \\
2\left\{x_{i}, y_{i}\right\} & =-2 \sum_{l>i} x_{l} y_{l}-z^{2}, & & \text { for all } i .
\end{aligned}
$$

This is the quasiclassical limit of the odd dimensional quantum Euclidean space, $O_{q^{1 / 2}}^{2 N-1}(\mathbb{C})$, introduced by Reshetikhin, Takhtadzhyan, and Faddeev, [18, Definition 12]. (See [16, $\S \S 2.1,2.2]$ for a simplified set of relations.)

5.5. $C_{N+1}$ case. In this case, $\mathfrak{g} \cong \mathfrak{s p}_{2 N}$ and

$$
\theta=2 \alpha_{1}+\ldots+2 \alpha_{N-1}+\alpha_{N}=2 \omega_{1} .
$$

The only cominuscule root is $\alpha_{N}$, and $\mathfrak{l}_{\Gamma \backslash\left\{\alpha_{N}\right\}}^{\prime} \cong \mathfrak{s l}_{N}$. Then under the adjoint action the nilradical $\mathfrak{n}_{\Gamma \backslash\left\{\alpha_{N}\right\}}^{+}$corresponds to the second symmetric power $S^{2}\left(V_{\mathfrak{s l}_{N}}^{\omega_{1}}\right) \cong$ $V_{\mathfrak{s l}_{N}}^{2 \omega_{1}}$ of the vector representation of $\mathfrak{s l}_{N}$. If we identify $\mathfrak{n}_{\Gamma \backslash\left\{\alpha_{N}\right\}}^{-}$with the space of symmetric matrices of size $N$ by

$$
\mathfrak{n}_{\Gamma \backslash\left\{\alpha_{N}\right\}}^{-}=\left\{\left[\begin{array}{cc}
0 & 0 \\
Y & 0
\end{array}\right] \mid Y=\left(y_{i j}\right)_{i, j=1}^{N}, y_{i j}=y_{j i}\right\},
$$


then the Poisson structure on $\mathfrak{n}_{\Gamma \backslash\left\{\alpha_{N}\right\}}^{-}$is given by

$$
\begin{aligned}
\left\{y_{i j}, y_{l m}\right\}=( & \operatorname{sign}(m-i)+\operatorname{sign}(l-j)) y_{i l} y_{j m} \\
& +(\operatorname{sign}(l-i)+\operatorname{sign}(m-j)) y_{i m} y_{j l} .
\end{aligned}
$$

Interestingly, this Poisson structure is not, as one might expect after seeing $\S 5.4$, the quasiclassical limit of a quantum symplectic space (see [18, Definition 14] or [16. $\S 1.1])$. Instead, it is the quasiclassical limit of the algebra of quantum symmetric matrices introduced by Noumi in [17. Theorem 4.3, Proposition 4.4, and comments following the proof] (with the parameters $a_{k}$ all set equal to 1), and of the one given by Kamita [11, Theorem 0.2] (with $q$ and $q^{-1}$ interchanged).

5.6. $D_{N}$ case. In this case, $\mathfrak{g}=\mathfrak{s o}_{2 N}$,

$$
\theta=\alpha_{1}+2 \alpha_{2}+\ldots+2 \alpha_{N-2}+\alpha_{N-1}+\alpha_{N}=\omega_{2},
$$

and the cominuscule roots are $\alpha_{1}, \alpha_{N-1}$, and $\alpha_{N}$. (We will assume $N \geq 4$.) Below we consider those three cases.

(a) Root $\alpha_{1}$. Here $\mathfrak{l}_{\Gamma \backslash\left\{\alpha_{1}\right\}}^{\prime} \cong \mathfrak{s o}_{2(N-1)}$ and $\mathfrak{n}_{\Gamma \backslash\left\{\alpha_{1}\right\}}^{-}$, considered an $\mathfrak{l}_{\Gamma \backslash\left\{\alpha_{1}\right\}^{-}}^{\prime}$ module under the adjoint action, is isomorphic to the vector representation $V_{\mathfrak{s o}_{2}(N-1)}^{\omega_{1}}$ of $\mathfrak{s o}_{2(N-1)}$. We identify

$$
\begin{aligned}
\mathfrak{n}_{\Gamma \backslash\left\{\alpha_{1}\right\}}^{-} & =\left\{\sum_{i=2}^{N} x_{i}\left(E_{N+i, 1}-E_{N+1, i}\right)+\sum_{j=2}^{N} y_{j}\left(E_{j, 1}-E_{N+1, N+j}\right) \mid x_{i}, y_{j} \in \mathbb{C}\right\} \\
& =\mathbb{A}^{2(N-1)} .
\end{aligned}
$$

Then the induced Poisson structure on $\mathfrak{n}_{\Gamma \backslash\left\{\alpha_{1}\right\}}^{-}$is given by

$$
\begin{aligned}
2\left\{x_{i}, x_{j}\right\} & =x_{i} x_{j}, & & i<j, \\
2\left\{y_{i}, y_{j}\right\} & =-y_{i} y_{j}, & & i<j, \\
2\left\{x_{i}, y_{j}\right\} & =x_{i} y_{j}, & & i \neq j, \\
\left\{x_{i}, y_{i}\right\} & =-\sum_{l>i} x_{l} y_{l}, & & \text { for all } i .
\end{aligned}
$$

It is the quasiclassical limit of the even dimensional quantum Euclidean space $O_{q^{1 / 2}}^{2(N-1)}(\mathbb{C}) ;$ see [18], [16].

(b) Root $\alpha_{N}$. In this case $\mathfrak{l}_{\Gamma \backslash\left\{\alpha_{N}\right\}}^{\prime} \cong \mathfrak{s l}_{N}$, and under the adjoint action $\mathfrak{n}_{\Gamma \backslash\left\{\alpha_{N}\right\}}^{+}$ is isomorphic to $V_{\mathfrak{s l}_{N}}^{\omega_{2}} \cong \bigwedge^{2} V_{\mathfrak{s l}_{N}}^{\omega_{1}}$, where $V_{\mathfrak{s l}_{N}}^{\omega_{1}}$ is the vector representation of $\mathfrak{s l}_{N}$. Identify $\mathfrak{n}_{\Gamma \backslash\left\{\alpha_{N}\right\}}^{-}$with the space of skew-symmetric matrices of size $N$ by

$$
\mathfrak{n}_{\Gamma \backslash\left\{\alpha_{N}\right\}}^{-}=\left\{\left[\begin{array}{cc}
0 & 0 \\
Y & 0
\end{array}\right] \mid Y=\left(y_{i j}\right)_{i, j=1}^{N}, y_{i j}=-y_{j i}\right\} .
$$

Then the Poisson structure on $\mathfrak{n}_{\Gamma \backslash\left\{\alpha_{N}\right\}}^{-}$is given by

$$
\begin{aligned}
2\left\{y_{i j}, y_{l m}\right\}= & (\operatorname{sign}(l-j)+\operatorname{sign}(m-i)) y_{i l} y_{j m} \\
& -(\operatorname{sign}(l-i)+\operatorname{sign}(m-j)) y_{i m} y_{j l} .
\end{aligned}
$$

This is the quasiclassical limit of the algebra of quantum antisymmetric matrices introduced by Strickland in [22, Section 1] (with $q$ replaced by $q^{1 / 2}$ ). 
(c) Root $\alpha_{N-1}$. One can lift the involutive automorphism of the Dynkin graph $D_{N}$ (preserving $\alpha_{1}, \ldots, \alpha_{N-2}$, and interchanging $\alpha_{N-1}$ and $\alpha_{N}$ ) to an automorphism of $\mathfrak{s o}_{2 N}$ that restricts to an isomorphism between $\mathfrak{l}_{\Gamma \backslash\left\{\alpha_{N-1}\right\}}$ and $\mathfrak{l}_{\Gamma \backslash\left\{\alpha_{N}\right\}}$ (interchanging their $r$-matrices) and their modules $\mathfrak{n}_{\Gamma \backslash\left\{\alpha_{N-1}\right\}}^{-}$and $\mathfrak{n}_{\Gamma \backslash\left\{\alpha_{N}\right\}}^{-}$. As a result, that automorphism of $\mathfrak{s o}_{2 N}$ restricts to an isomorphism between the Poisson structures on $\mathfrak{n}_{\Gamma \backslash\left\{\alpha_{N-1}\right\}}^{-}$and $\mathfrak{n}_{\Gamma \backslash\left\{\alpha_{N}\right\}}^{-}$.

5.7. $E_{6}$ case. With this example, we show that compact Hermitian symmetric spaces for exceptional groups give rise to new interesting quadratic Poisson structures on affine spaces.

The highest root of the simple Lie algebra $\mathfrak{e}_{6}$ of type $E_{6}$ is

$$
\theta=\alpha_{1}+2 \alpha_{2}+2 \alpha_{3}+3 \alpha_{4}+2 \alpha_{5}+\alpha_{6}=\omega_{2},
$$

and $\mathfrak{e}_{6}$ has two cominuscule roots: $\alpha_{1}$ and $\alpha_{6}$. Similarly to $\S 5.6(\mathrm{c})$, one lifts the involutive automorphism of the Dynkin graph $E_{6}$ that interchanges $\alpha_{1}$ and $\alpha_{6}$ and fixes the other nodes to an automorphism of $\mathfrak{e}_{6}$ that restricts to an isomorphism between $\mathfrak{l}_{\Gamma \backslash\left\{\alpha_{1}\right\}}^{\prime}$ and $\mathfrak{l}_{\Gamma \backslash\left\{\alpha_{6}\right\}}^{\prime}$, and between their modules $\mathfrak{n}_{\Gamma \backslash\left\{\alpha_{1}\right\}}^{-}$and $\mathfrak{n}_{\Gamma \backslash\left\{\alpha_{6}\right\}}^{-}$. This linear map provides an isomorphism between the induced Poisson structures on $\mathfrak{n}_{\Gamma \backslash\left\{\alpha_{1}\right\}}^{-}$and $\mathfrak{n}_{\Gamma \backslash\left\{\alpha_{6}\right\}}^{-}$.

In the case of the root $\alpha_{1}$, we have $\mathfrak{I}_{\Gamma \backslash\left\{\alpha_{1}\right\}}^{\prime} \cong \mathfrak{s o}_{10}$, and as an $\mathfrak{I}_{\Gamma \backslash\left\{\alpha_{1}\right\}}^{\prime}$-module $\mathfrak{n}_{\Gamma \backslash\left\{\alpha_{1}\right\}}^{+}$is isomorphic to one of the half-spin representations $V_{\mathfrak{s o}_{10}}^{\omega_{5}}$ of $\mathfrak{s o}_{10}$. As a vector space, $V_{\mathfrak{s o}_{10}}^{\omega_{5}}$ is identified with

$$
V \oplus \bigwedge^{3} V \oplus \bigwedge^{5} V
$$

for a 5 dimensional vector space $V$; cf. [9, Section 20]. A basis $\left\{v_{1}, \ldots, v_{5}\right\}$ of $V$ gives rise to the basis $\left\{v_{i_{1}} \wedge \cdots \wedge v_{i_{n}} \mid n\right.$ odd, $\left.i_{1}<\ldots<i_{n}\right\}$ of $V_{\mathfrak{s 0}_{10}}^{\omega_{5}}$. We view it as a set of coordinate functions

$$
\left\{y_{I}|I \subset\{1, \ldots, 5\},| I \mid \text { odd }\right\}
$$

on $\left(V_{\mathfrak{s 0}_{10}}^{\omega_{5}}\right)^{*}$. In terms of those coordinates, the induced quadratic Poisson structure on this 16 dimensional affine space is given by

$$
\begin{aligned}
\left\{y_{I}, y_{J}\right\}= & \sum_{\substack{i \in I \backslash J \\
j \in J \backslash I \\
i \neq j}} \operatorname{sign}(j-i) a_{i, j}^{I, J} y_{(I \backslash\{i\}) \cup\{j\}} y_{(J \backslash\{j\}) \cup\{i\}} \\
& -\frac{1}{2} \sum_{\substack{\{i, j\} \subset I \backslash J \\
i<j}} a_{i, j}^{I, J} y_{I \backslash\{i, j\}} y_{J \cup\{i, j\}}+\frac{1}{2} \sum_{\substack{\{i, j\} \subset J \backslash I \\
i<j}} a_{i, j}^{I, J} y_{I \cup\{i, j\}} y_{J \backslash\{i, j\}} .
\end{aligned}
$$

Here, for two subsets $I$ and $J$, and two elements $i$ and $j$ of $\{1, \ldots, 5\}$, we set

$$
I(i, j)=\{l \in I \mid i<l<j \text { or } j<l<i\}
$$

and

$$
a_{i, j}^{I, J}=(-1)^{|I(i, j)|+|J(i, j)|} .
$$

For example, for $i<j$,

$$
\left\{y_{\{i\}}, y_{\{j\}}\right\}=y_{\{i\}} y_{\{j\}} \text {. }
$$




\section{ACKNOWLEDGEMENTS}

We thank T. H. Lenagan, J.-H. Lu, K. Rietsch, R. Steinberg, and J. Wolf for helpful discussions and correspondence. We also thank the referee for the careful reading of the manuscript and for pointing out typos in Section 4.

\section{REFERENCES}

1. N. Bourbaki, Groupes et algèbres de Lie, Chaps. 4, 5 et 6, Hermann, 1975. MR 0453824 (56:12077)

2. M. Brion and V. Lakshmibai, A geometric approach to standard monomial theory, Represent. Theory 7 (2003), 651-680. MR 2017071 (2004m:14106)

3. K. A. Brown, K. A. Goodearl, and M. Yakimov, Poisson structures on affine spaces and flag varieties. I. Matrix affine Poisson space, Advances in Math. 206 (2006), 567-629. MR.2263715 (2007k:14094)

4. R. W. Carter, Finite Groups of Lie Type. Conjugacy Classes and Complex Characters, WileyInterscience, Chichester, 1993. MR1266626 (94k:20020)

5. M. Crainic and R. L. Fernandes, Integrability of Poisson brackets, J. Diff. Geom. 66 (2004), 71-137. MR2128714 (2006f:53119)

6. V. Deodhar, On some geometric aspects of Bruhat orderings. I. A finer decomposition of Bruhat cells, Invent. Math. 79 (1985), 499-511. MR782232 (86f:20045)

7. V. G. Drinfeld, On Poisson homogeneous spaces of Poisson-Lie groups, Theor. and Math. Phys. 95 (1993), 524-525. MR1243249 (94k:58045)

8. P. Foth and J.-H. Lu, A Poisson structure on compact symmetric spaces, Comm. Math. Phys. 251 (2004), 557-566. MR2102330 (2005g:53156)

9. W. Fulton and J. Harris, Representation Theory, A First Course, Graduate Texts in Math. 129, Springer-Verlag, Berlin, 1991. MR1153249 (93a:20069)

10. K. R. Goodearl, Prime spectra of quantized coordinate rings, in Interactions Between Ring Theory and Representations of Algebras (Murcia 1998), Lecture Notes in Pure and Appl. Math., 210, Dekker, New York, 2000, pp. 205-237. MR1759846 (2001b:16052)

11. A. Kamita, Quantum deformations of certain prehomogeneous vector spaces III, Hiroshima Math. J. 30 (2000), 79-115. MR.1753385 (2001a:17021)

12. D. Kazhdan and G. Lusztig, Representations of Coxeter groups and Hecke algebras, Invent. Math. 53 (1979), 165-184. MR560412 (81j:20066)

13. J.-H. Lu and M. Yakimov, Partitions of the wonderful group compactification, Transformation Groups, 12 (2007), no. 4, 695-723. MR2365441

14. G. Lusztig, Total positivity in partial flag manifolds, Representation Theory 2 (1998), 70-78. MR.1606402 (2000b:20060)

15. I. Muller, H. Rubenthaller, and G. Schiffmann, Structure des espaces prèhomogènes associès á certaines algèbres de Lie graduèes, Math. Ann. 274 (1986), 95-123. MR834108(88e:17025)

16. I. M. Musson, Ring theoretic properties of the coordinate rings of quantum symplectic and Euclidean space, in Ring Theory, Proc. Biennial Ohio State-Denison Conf., 1992 (S. K. Jain and S. T. Rizvi, eds.), World Scientific, Singapore, 1993, pp. 248-258. MR 1344235(96e:16052)

17. M. Noumi, Macdonald's symmetric polynomials as zonal spherical functions on some quantum homogeneous spaces, Advances in Math. 123 (1996), 16-77. MR.1413836 (98a:33004)

18. N. Yu. Reshetikhin, L. A. Takhtadzhyan, and L. D. Faddeev, Quantization of Lie groups and Lie algebras, Leningrad Math. J. 1 (1990), 193-225. MR1015339 (90j:17039)

19. R. Richardson, G. Röhrle, and R. Steinberg, Parabolic subgroups with Abelian unipotent radical, Invent. Math. 110 (1992), 649-671. MR1189494 (93j:20092)

20. K. Rietsch, Closure relations for totally nonnegative cells in $G / P$, Math. Res. Lett. 13 (2006), 775-786. MR2280774 (2007j:14073)

21. T. A. Springer, Intersection cohomology of $B \times B$-orbit closures in group compactifications, with an appendix by W. van der Kallen. Special issue in celebration of Claudio Procesi's 60th birthday, J. Algebra 258 (2002), 71-111. MR1958898 (2004a:14025)

22. E. Strickland, Classical invariant theory for the quantum symplectic group, Advances in Math. 123 (1996), 78-90. MR1413837 (98a:17022)

23. M. Takeuchi, On orbits in a compact hermitian symmetric space, Am. J. Math. 90 (1968), 657-680. MR0245827(39:7133) 
24. J. A. Wolf, Fine structure of Hermitian symmetric spaces, in Symmetric Spaces, Short Courses Presented at Washington University (W. Boothby, G. L. Weiss, eds.), New York, Marcel Dekker, 1972, pp. 271-357. MR0404716 (53:8516)

25. J. A. Wolf, Classification and Fourier inversion for parabolic subgroups with square integrable nilradical, Mem. Amer. Math. Soc. 225 (1979), 1-166. MR.546511(81a:22010)

26. P. Xu, Dirac submanifolds and Poisson involutions, Ann. Sci. École Norm. Sup. (4) 36 (2003), 403-430. MR1977824 (2004e:53125)

Department of Mathematics, University of California, Santa Barbara, California 93106

E-mail address: goodearl@math.ucsb.edu

Department of Mathematics, University of California, Santa Barbara, California 93106

E-mail address: yakimov@math.ucsb.edu 